\title{
BMJ Open Improving risk factor management for patients with poorly controlled type 2 diabetes: a systematic review of healthcare interventions in primary care and community settings
}

\author{
Mark E Murphy, ${ }_{1}^{1}$ Molly Byrne, ${ }^{2}$ Rose Galvin, ${ }^{3}$ Fiona Boland, ${ }^{1}$ Tom Fahey, ${ }^{1}$ \\ Susan M Smith ${ }^{1}$
}

To cite: Murphy ME, Byrne M, Galvin R, et al. Improving risk factor management for patients with poorly controlled type 2 diabetes: a systematic review of healthcare interventions in primary care and community settings. BMJ Open 2017;7:e015135. doi:10.1136/ bmjopen-2016-015135

- Prepublication history and additional material for this paper are available online. To view these files please visit the journal online (http://dx.doi. org/10.1136/bmjopen-2016015135).

Received 11 November 2016 Revised 18 May 2017 Accepted 31 May 2017

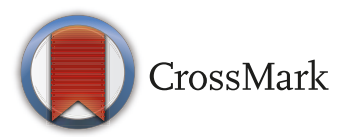

${ }^{1}$ Department of General Practice, HRB Centre for Primary Care Research, Royal College of Surgeons, Dublin, Ireland ${ }^{2}$ Department of Physiotherapy, University of Limerick, Ireland ${ }^{3}$ Health Behaviour Change Research Group, School of Psychology, National University of Ireland, Galway, Ireland

Correspondence to Dr Mark E Murphy; markmurphy@rcsi.ie

\section{ABSTRACT}

Objectives Poorly controlled type 2 diabetes mellitus (T2DM) is a major international health problem. Our aim was to assess the effectiveness of healthcare interventions, specifically targeting patients with poorly controlled T2DM, which seek to improve glycaemic control and cardiovascular risk in primary care settings.

Design Systematic review.

Setting Primary care and community settings. Included studies Randomised controlled trials (RCTs) targeting patients with poor glycaemic control were identified from Pubmed, Embase, Web of Science, Cochrane Library and SCOPUS. Poor glycaemic control was defined as HbA1c over $59 \mathrm{mmol} / \mathrm{mol}(7.5 \%)$.

Interventions Interventions were classified as organisational, patient-oriented, professional, financial or regulatory.

Outcomes Primary outcomes were $\mathrm{HbA} 1 \mathrm{c}$, blood pressure and lipid control. Two reviewers independently assessed studies for eligibility, extracted data and assessed study quality. Meta-analyses were undertaken where appropriate using random-effects models. Subgroup analysis explored the effects of intervention type, baseline $\mathrm{HbA1c}$, study quality and study duration. Meta-regression analyses were undertaken to investigate identified heterogeneity.

Results Forty-two RCTs were identified, including 11250 patients, with most undertaken in USA. In general, studies had low risk of bias. The main intervention types were patient-directed (48\%) and organisational (48\%). Overall, interventions reduced $\mathrm{HbA} 1 \mathrm{c}$ by $-0.34 \%(95 \% \mathrm{Cl}-0.46 \%$ to $-0.22 \%$ ), but meta-analyses had high statistical heterogeneity. Subgroup analyses suggested that organisational interventions and interventions on those with baseline $\mathrm{HbA1c}$ over $9.5 \%$ had better improvements in $\mathrm{HbA1C}$. Meta-regression analyses suggested that only interventions on those with population $\mathrm{HbA1c}$ over $9.5 \%$ were more effective. Interventions had a modest improvement of blood pressure and lipids, although baseline levels of control were generally good.

Conclusions This review suggests that interventions for T2DM, in primary care, are better targeted at individuals with very poor glycaemic control and that organisational interventions may be more effective.
Strengths and limitations of the study

- This systematic review adds to the evidence regarding the effectiveness of healthcare interventions, which specifically target patients with poor glycaemic control of type 2 diabetes mellitus, in community settings.

- There is no specific definition for 'poor control' diabetes in the literature, but by including all studies that had patients with a $\mathrm{HbA} 1 \mathrm{c} \geq 59 \mathrm{mmol} / \mathrm{mol}$ (7.5\%), we captured the full range of poor glycaemic control and also examined other key risk factors such as blood pressure and lipids.

- Data were pooled from 42 studies across four continents, enhancing the generalisability of the findings.

- We did not account for medication use in the studies, but given that all included studies were randomised controlled trials, which would balance out delivery of medications, we think that differences in underlying medication usage may relate to how different interventions promote intensification of medications.

- An individual patient data meta-analysis may answer further questions not possible in this review.

\section{INTRODUCTION}

Worldwide, type 2 diabetes mellitus (T2DM) is rising in prevalence and will exceed $4.4 \%$ of the world's population or 366 million by 2030. ${ }^{1}$ Despite a wealth of evidence regarding the importance of risk factor control in T2DM, many patients continue to have poor control of HbAlc, blood pressure and lipids. Up to $60 \%$ of the patients fail to meet target HbA1c levels. ${ }^{2}$ Similarly, over one-third of the patients with T2DM have inadequate blood pressure control. ${ }^{3}$ Poorly controlled T2DM-and its associated microvascular and macrovascular complications-is associated with higher morbidity, higher mortality, poorer quality of life and substantial economic burden. ${ }^{4}$ 
Several studies have examined interventions designed to support the delivery of diabetes care in the community to improve glycaemic and cardiovascular risk factor control. ${ }^{5-11}$ A 2011 review of community-based interventions including all patients with T2DM, comprising 68 studies, showed that only one-third had a statistically significant improvement in one of the relevant clinical outcomes for diabetes: HbAlc, blood pressure or lipids. ${ }^{8}$ The majority of the included studies targeted all patients with T2DM without focussing on those with poor control. Although no overall effect was noted, combining organisational with professional (multifaceted) interventions was concluded to be more beneficial than single interventions and the highest quality multifaceted randomised controlled trials (RCTs) tended to include decision support interventions and elements. A 2013 review looked at 48 cluster RCTs, assessing the effectiveness of quality improvement (QI) strategies on the management of diabetes (both T1DM and T2DM) ${ }^{11}$ It suggested that QI interventions, which intervened at a system level on diabetes management, were associated with the largest benefits in glycaemic control and that the effectiveness of interventions targeting healthcare practitioners varied with baseline glycaemic control, being more effective with patients with worse control. ${ }^{11}$ A 2016 review, of T1DM or T2DM in primary care, looked at the effects of Clinician Education, Clinician Reminders, Team Changes, Case Management, Electronic Patient Registry, Telemedicine and Audit and Feedback. ${ }^{10}$ Including 30 studies, it concluded that multifaceted interventions on multidisciplinary teams were most effective. Interventions targeting family physicians were only effective if computerised feedback on insulin prescribing was provided.

Four large RCTs from North America and the UK have investigated the effects of intensive management of hyperglycaemic and cardiac risk factors on mortality in T2DM across all settings. ${ }^{12-17}$ Uncertainty remains regarding intensive glycaemic management for all patients with T2DM, with concerns about aggressive reductions in HbAlc. ${ }^{18}$ Targeted reductions in cardiovascular and glycaemic risk factors in certain vulnerable populations (cognitively impaired, disabled and frail) have been advocated. ${ }^{19}$ Interventions that specifically target those with very poor control of risk factors may be more beneficial than those targeting all patients, achieving the benefits of cardiovascular and glycaemic control, but without the potential risks of intensively lowering HbA1c in all persons with T2DM. The effect of interventions specifically targeting patients with poorly controlled T2DM in primary care is unknown.

Our aim was to assess the effectiveness of healthcare interventions delivered in primary care and community settings, targeting poorly controlled T2DM, which seek to improve glycaemic control, blood pressure and lipids.

\section{METHODS}

The Preferred Reporting Items for Systematic Reviews and Meta-Analyses (PRISMA) guidelines were used to standardise the conduct and reporting of the research and the protocol was registered on PROSPERO. ${ }^{20}$

\section{Data sources and searches}

We searched articles in all languages from the Cochrane Library, Pubmed, Embase, Web of Science and SCOPUS from 1990 to 31 December 2016. Reference lists of all included papers were searched. Secondary searching of all references from included studies was also conducted. Online supplementary appendix 1 outlines the search string.

\section{Study selection}

We considered RCTs, controlled clinical trials, controlled before and after studies and interrupted time series analyses meeting the Cochrane Effective Practice and Organisation of Care (EPOC) quality criteria. ${ }^{21}$ Studies published in all languages were eligible.

\section{Population}

Individuals with 'poorly controlled' T2DM were our population of interest. Though there is a broad consensus about the importance of achieving good glycaemic control for the reasons described, there are no validated cut-offs, which define 'poor-control' of T2DM for targeted interventions. Poorly controlled T2DM has been defined based on elevated glycated haemoglobin levels in the literature, with different thresholds of HbAlc described, from over $59 \mathrm{mmol} / \mathrm{mol}(7.5 \%)$, over $64 \mathrm{mmol} / \mathrm{mol}$ $(8.0 \%)$ to over $75 \mathrm{mmol} / \mathrm{mol}(9.0 \%) .{ }^{22-24}$ In this review, we considered participants to have poorly controlled T2DM if their HbAlc was over $59 \mathrm{mmol} / \mathrm{mol}(7.5 \%$ ) (or if over $80 \%$ of the population in a study had a HbAlc over $59 \mathrm{mmol} / \mathrm{mol}$ ). Similarly, there is no defined cut-off as to what defines 'poorly controlled' blood pressure. We identified studies primarily based on poor glycaemic control and also included participants in these studies who had uncontrolled hypertension or elevated cholesterol/ lipids, if the risk factor level was above that of an accepted international target, as designated by the study authors. Where studies included patients with 'poor control' based on a range of risk factor profiles, for consistency, we only included a study if $80 \%$ of the population had a HbAlc over $59 \mathrm{mmol} / \mathrm{mol}(7.5 \%)$.

\section{Interventions}

We included interventions delivered by healthcare professionals specifically aiming to target patients with poor control of T2DM, based in primary care or community settings. The primary healthcare setting was defined as providing 'integrated, easy to access, healthcare services by clinicians who are accountable for addressing a large majority of personal healthcare needs, developing a sustained and continuous relationship with patients and practicing in the context of family and community'. ${ }^{25} \mathrm{We}$ excluded drug trials though interventions could have involved treatment intensification. Interventions were 
defined as simple if they had one identifiable component and multifaceted if they had more than one element. We excluded trials performed within the hospital or the hospital-outpatient setting. The Cochrane EPOC taxonomy of interventions was used and the predominant intervention type was defined using five categories including organisational, patient-centred, regulatory, financial and professional. Examples of these intervention types are provided in online supplementary appendix $2 .^{21}$

\section{Comparison}

Comparison groups were included if they received usual care in that setting for T2DM. Controls were also included if they received minor enhanced elements of care, such as education leaflets, which the study authors believed did not go beyond usual care in most settings.

\section{Outcome measures}

Primary outcomes included glycaemic control (HbA1c), blood pressure (systolic or diastolic) and lipid levels, but if studies did not include HbAlc, they were excluded. Secondary outcomes included patient-reported outcome measures (PROMs) (eg, health-related quality of life), utilisation of health services, behavioural outcomes such as medication adherence, provider behaviour, acceptability of service to patients and providers, economic outcomes and adverse events.

\section{Data extraction and quality assessment}

Two reviewers (MEM and RG) read the titles and/or abstracts of the identified references and eliminated irrelevant studies. Studies that were deemed eligible for inclusion were read in full and their suitability for inclusion in the systematic review was independently determined by two reviewers. Disagreements were managed by a third, independent reviewer (SMS). The following information was extracted: (a) details of intervention, (b) participants, (c) clinical setting, (d) study design, (e) outcomes, (f) author information. We contacted authors for missing data.

Risk of bias in articles was assessed using the Cochrane Handbook for systematic reviewing and EPOC criteria. ${ }^{26}$ Two review authors independently assessed the risk of bias of each included study against the criteria described in the Cochrane risk of bias tool. We explicitly judged each of these criteria using: low risk of bias, high risk of bias or unclear risk of bias (either lack of information or uncertainty over the potential for bias). We resolved disagreements by consensus and consulted a third review author to resolve disagreements if necessary. An overall assessment of a study's risk of bias was determined using EPOC guidance, with judgement and consensus reached between two reviewers (MEM and SMS). ${ }^{26}$

\section{Data analysis}

For continuous data, we calculated the treatment effect using mean differences (MDs) and 95\% CIs. No binary outcomes were included. Revman software was used to perform the analysis, determine heterogeneity and produce forest plots to illustrate pooled estimates. ${ }^{21}$
Stata version 13 was used to investigate publication bias by creating funnel plots and using Egger's test to assess funnel plot asymmetry. ${ }^{27} \mathrm{~A}$ random-effects analysis was performed and heterogeneity across the studies was quantified using the $\mathrm{I}^{2}$ statistic. The $\mathrm{I}^{2}$ statistic describes the percentage of the variability in effect estimates which is due to heterogeneity rather than sampling error (chance).$^{28}$ If the $\mathrm{I}^{2}$ statistic was $>50 \%$, it was deemed that there was significant heterogeneity between the studies.

Subgroup analyses were performed for primary outcomes based on a priori assumptions, as per the PROSPERO protocol..$^{20}$ For HbAlc, we explored the possible effects of subgroups: (a) the type of intervention based on the EPOC taxonomy (see online supplementary appendix 2); (b) study quality and (c) baseline HbAlc in the study populations (HbAlc $7.5 \%-9.4 \%$ or $\geq 9.5 \%$ ). After reviewing, the included studies we also included study duration as a subgroup $(<12$ months or $\geq 12$ months), as a wide range in study duration was found. Subgroup analyses for systolic blood pressure (SBP) and diastolic blood pressure (DBP) explored the effects of intervention-type based on the EPOC taxonomy.

When important heterogeneity was identified, we investigated its causes using meta-regression. Meta-regression is an extension to subgroup analysis that allows the effect of continuous, as well as categorical, characteristics to be investigated.$^{29}$ Meta-regression was performed to explore the effects of: (a) study quality (using the overall assessment risk of bias); (b) study population characteristics (eg, gender, age and baseline HbA1c and SBP); (c) intervention type (EPOC taxonomy) and (d) study duration on the primary outcomes. ${ }^{29}$ Random effects meta-regression was performed using Stata version $13 .{ }^{27}$

\section{RESULTS}

Overall 18829 titles were screened and 42 full text articles met the inclusion criteria (figure 1: PRISMA flow diagram). All 42 studies were RCTs, encompassing 50 interventions in total, comprising 11250 patients. ${ }^{22-2430-68}$ No other eligible study designs were identified.

\section{Characteristics of studies}

Twenty-nine of the 42 studies were conducted in USA, 9 in Europe, 2 in Australia, 1 in Mexico and 1 in Israel. Follow-up of outcomes in the studies varied in length from $3^{53}$ to 36 months. ${ }^{46}$ The mean HbAlc at baseline across all studies was $9.5 \%$ (95\% CI $9.3 \%$ to $9.8 \%$ ). The mean age of patients in the studies was 58.0, varying from 47.9 (62) to 67.5 (41) partly reflecting different inclusion criteria (table 1 ). Thirty studies explicitly defined their study population as 'poorly controlled', 'complicated' or 'persistently poorly controlled', whereas the other 12 had poorly controlled T2DM with HbA1c $\geq 59 \mathrm{mmol} / \mathrm{mol}(7.5 \%)$ as per the review inclusion criteria. Twenty-seven of the 42 studies reported SBP results ${ }^{22-24} 30-36383941454648-515458-6062656668$ and of these, 23 reported DBP. $22-24313234-363839414546484951545859$ 62656668 Twenty of the studies reported a lipid outcome. ${ }^{23}$ 


\begin{tabular}{|c|c|c|}
\hline $\begin{array}{c}\text { Records identified through } \\
\text { Medline } \\
(n=2927)\end{array}$ & $\begin{array}{c}\text { Records identified through } \\
\text { Embase } \\
(n=3561)\end{array}$ & $\begin{array}{c}\text { Records identified through } \\
\text { Web of Science } \\
(n=9333)\end{array}$ \\
\hline
\end{tabular}

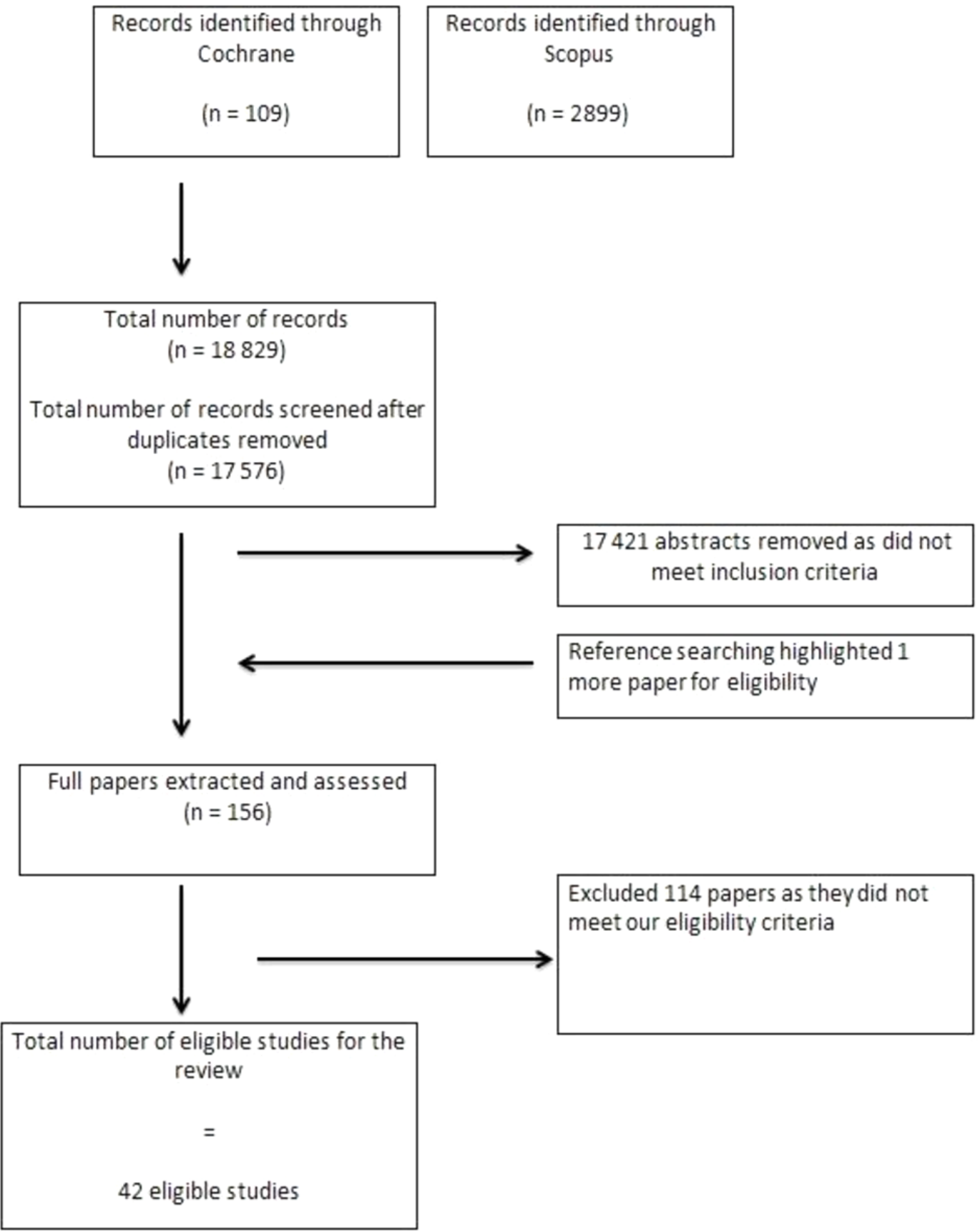

Figure 1 Preferred Reporting Items for Systematic Reviews and Meta-Analyses flow sheet.

$2430-32353638394145464851565862656668$ All of the 42 studies reported at least one secondary outcome. Two studies were excluded from primary outcome analysis due to lack of appropriate data, despite efforts to contact authors. ${ }^{3161}$

Interventions were all complex with multiple components. Studies were categorised based on the predominant intervention element using the EPOC taxonomy. The included interventions were categorised as predominantly patient-centred $(\mathrm{n}=20,48 \%)$, organisational $(\mathrm{n}=20,48 \%)$, financial $(n=1,2 \%)$ or professional $(n=1,2 \%)$. One study ${ }^{44}$ comprised two intervention arms with a patient-centred and financial intervention (included as a patient-centred predominant intervention in our analysis). Descriptions of the interventions are outlined in table 1.

The 20 patient-centred interventions in our review included 4 telephone-based, ${ }^{34} 4156585$ computerised/ 


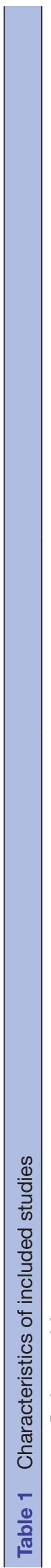

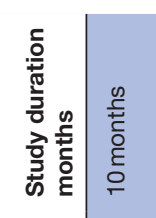

몬.
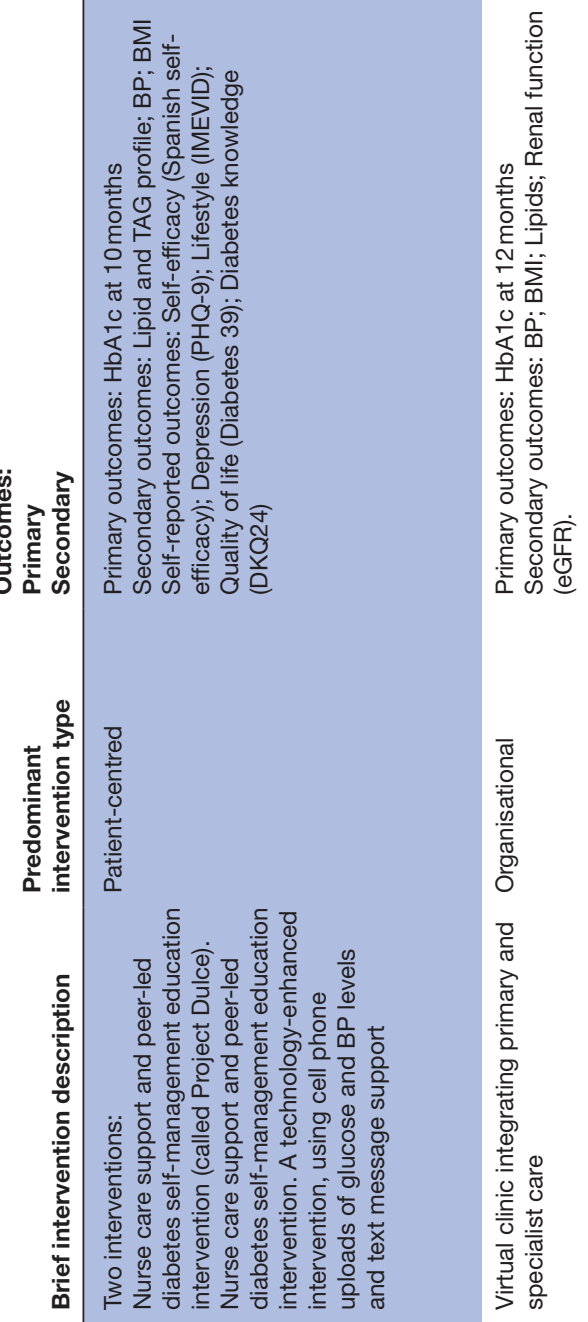

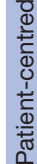

을

ㄸำ

)

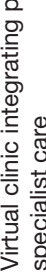

点

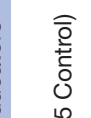

을

물

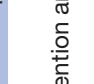

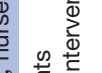

变

舟 $\frac{1}{2}$

১े

人ิm

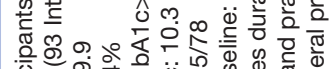

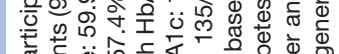

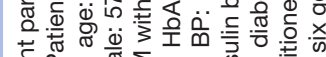

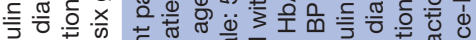

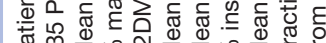

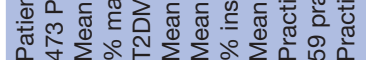
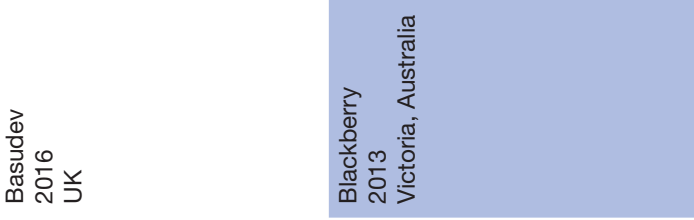


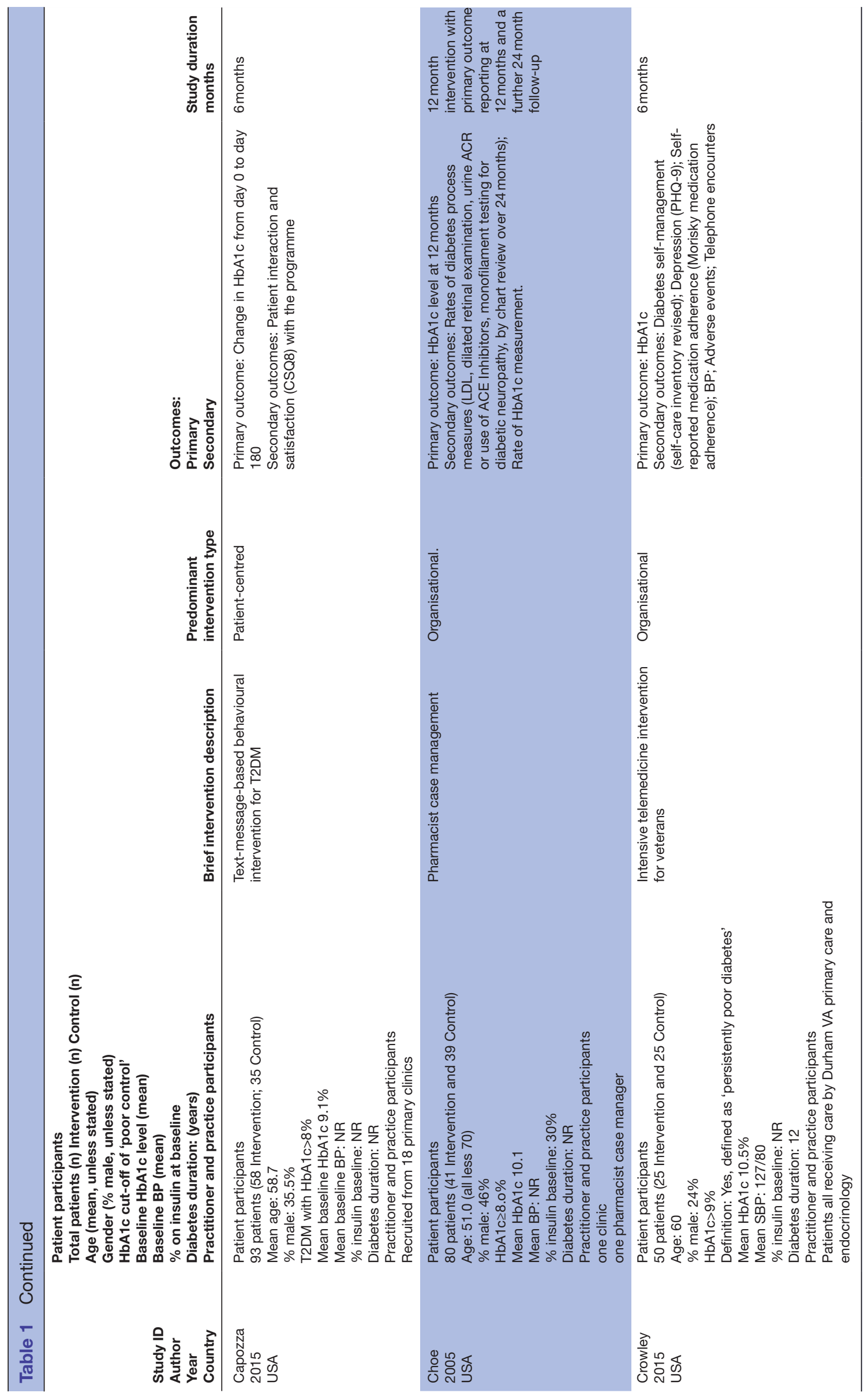




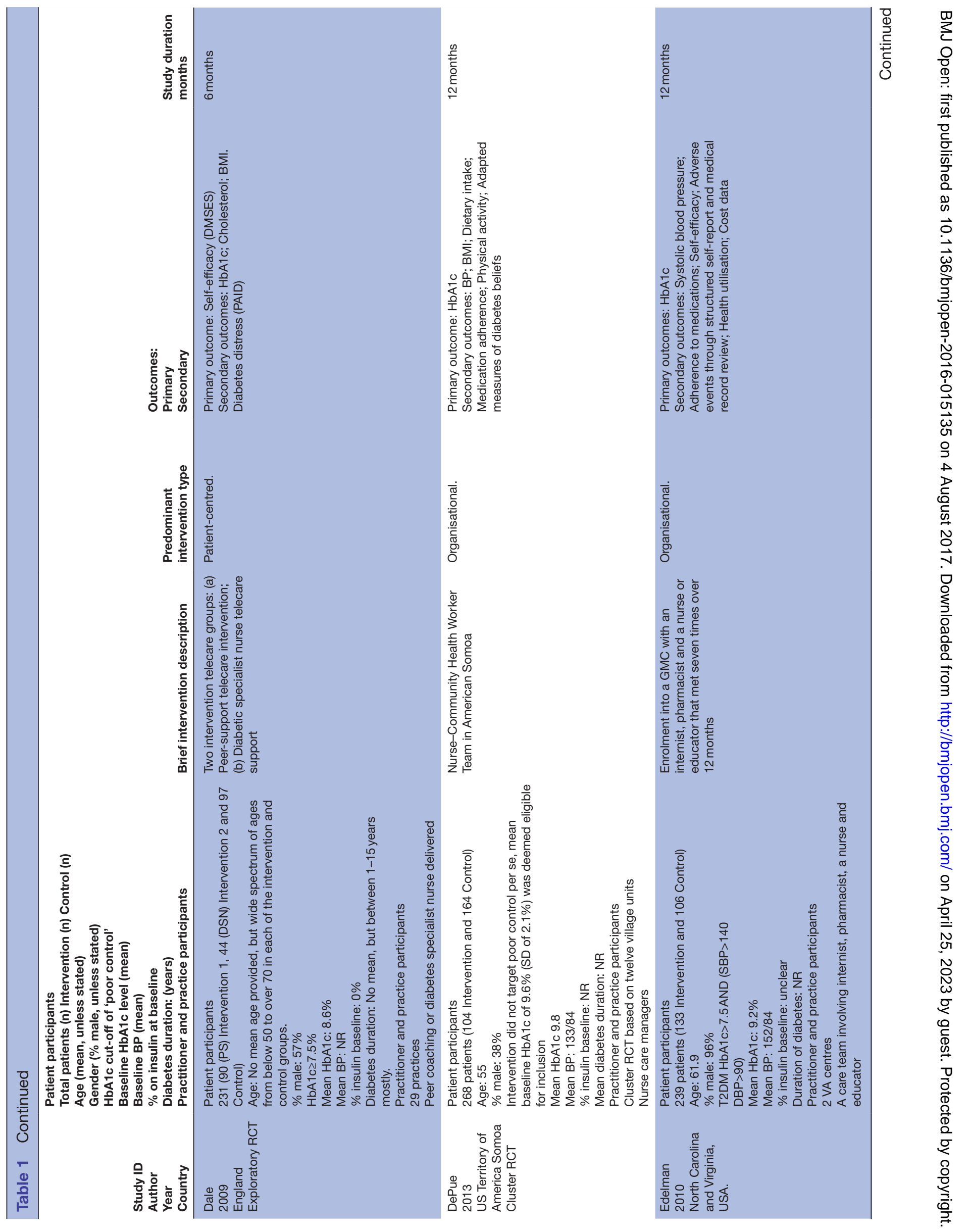




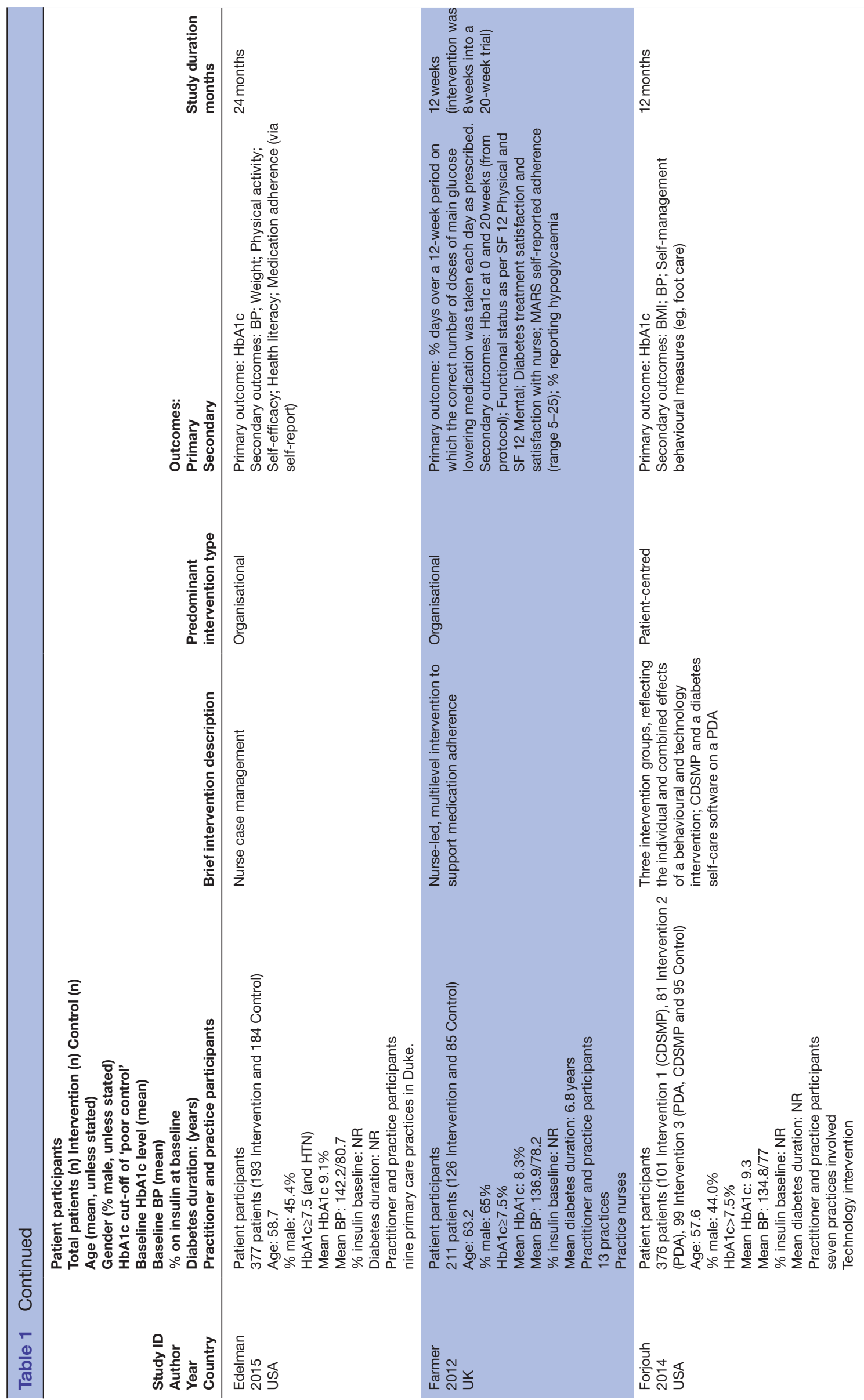




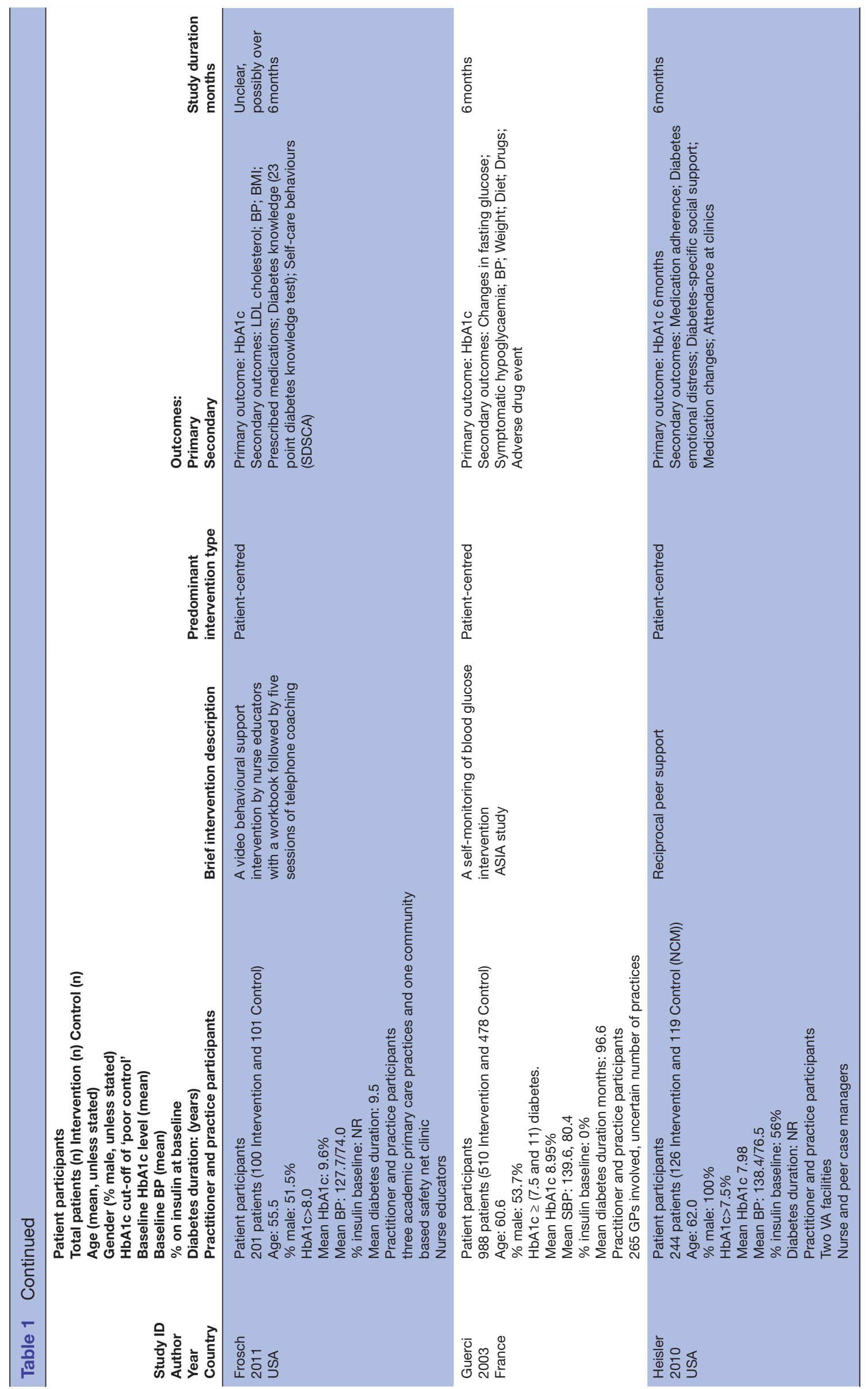



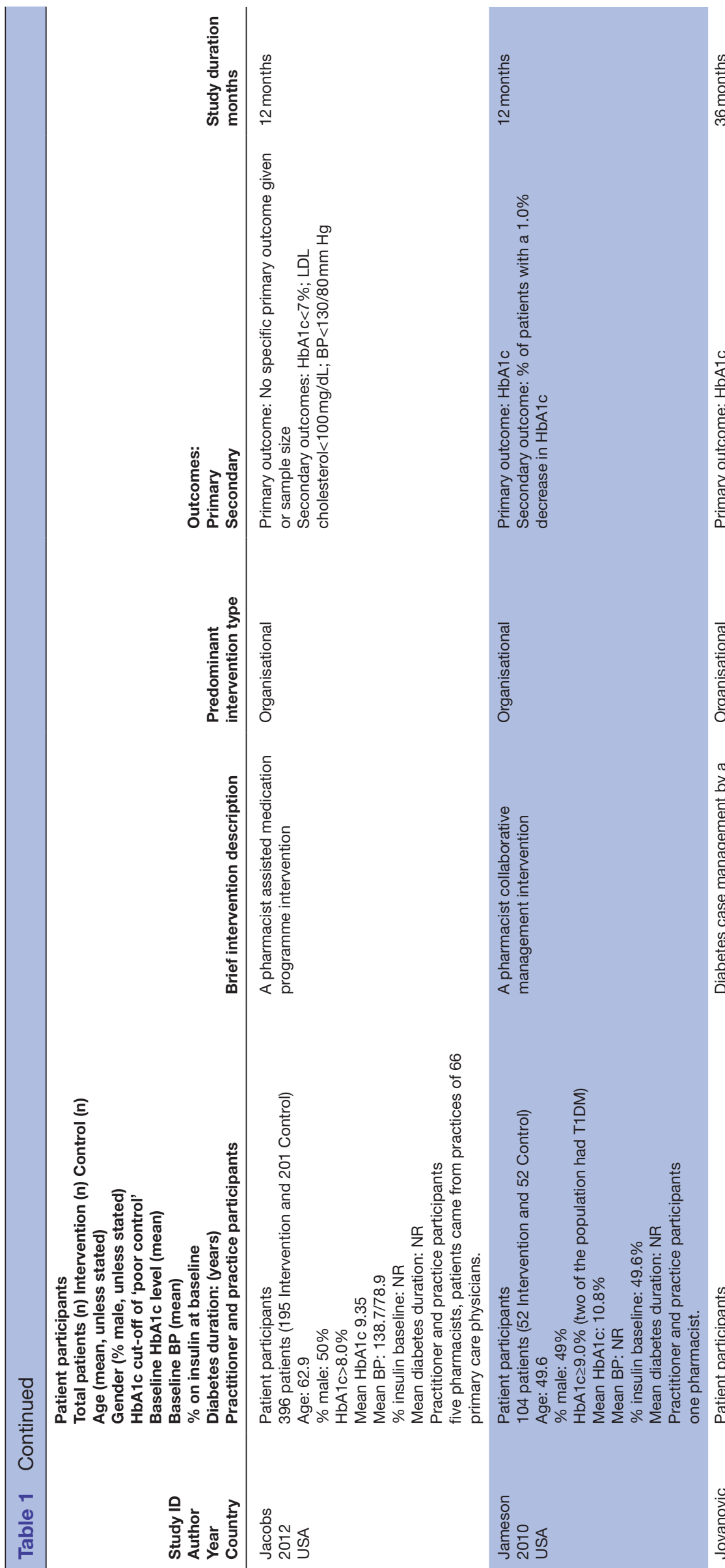

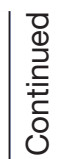

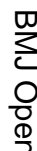
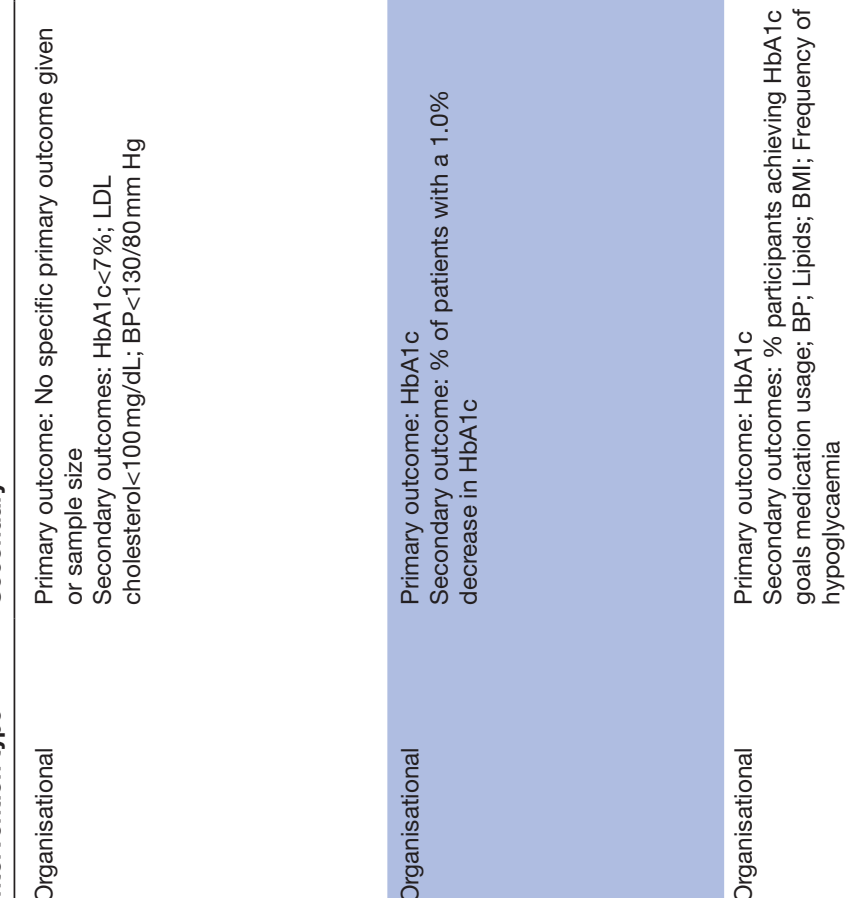

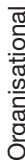
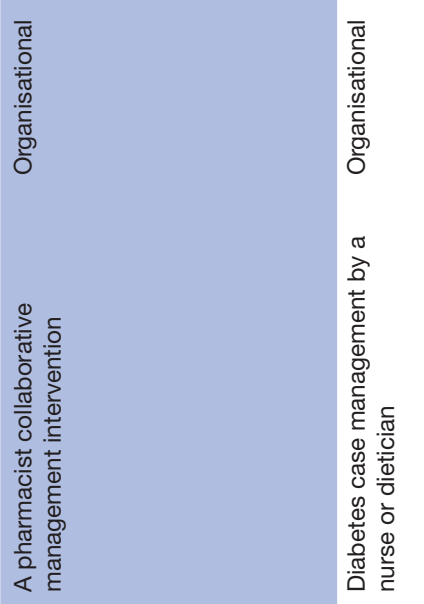


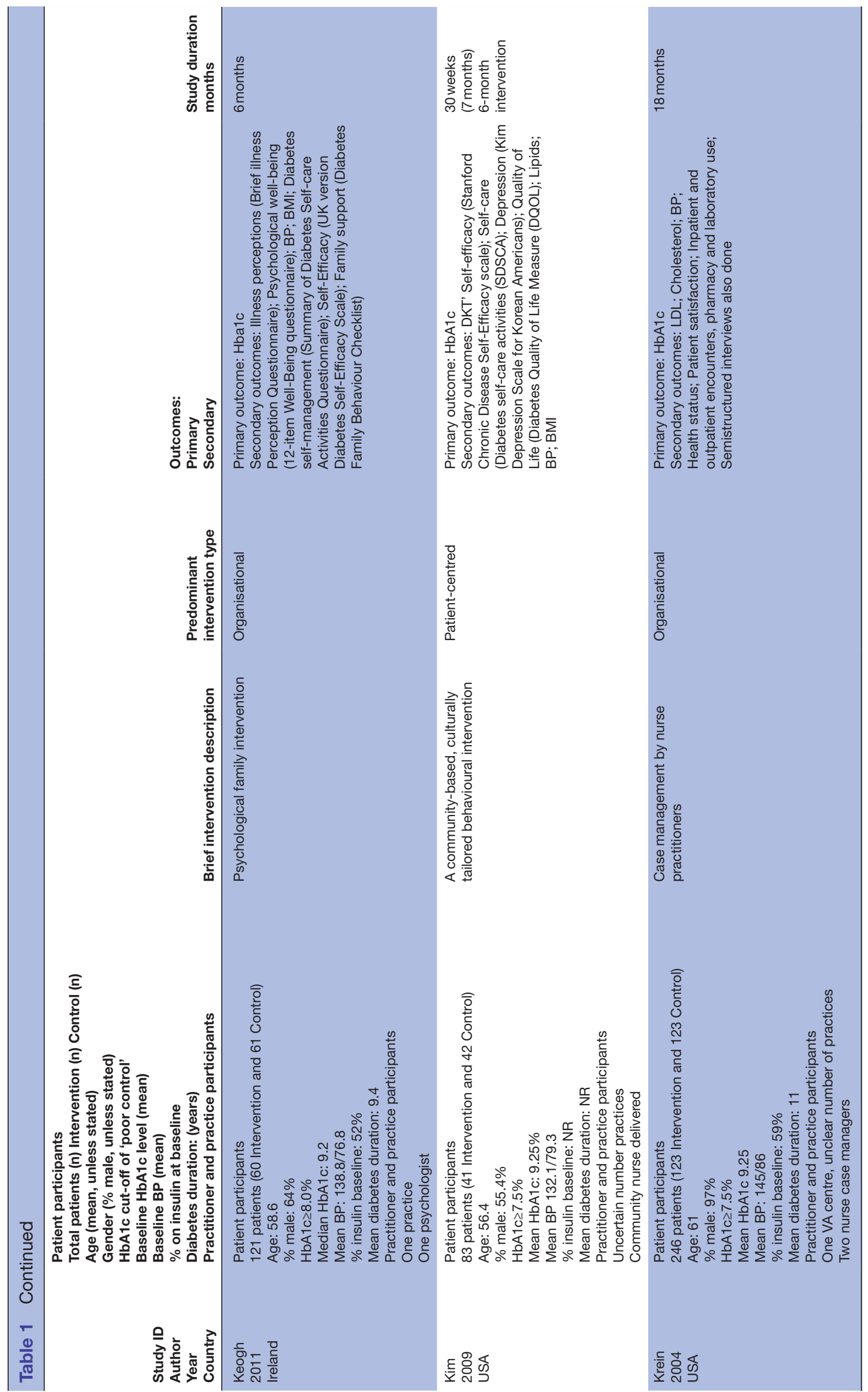



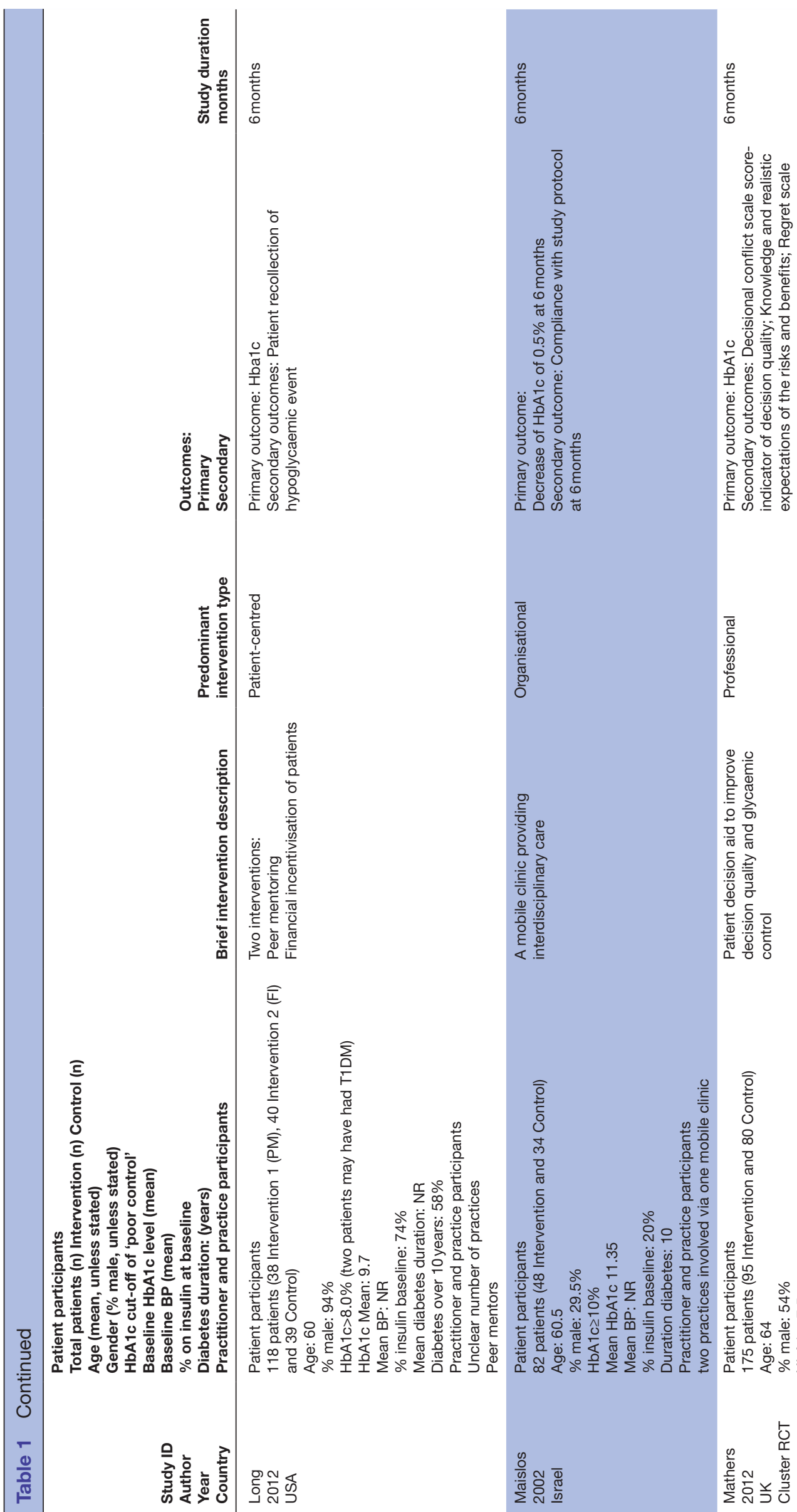

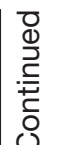

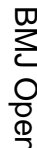

ज

응

$\frac{\overline{\bar{\sigma}}}{\overline{\mathrm{\sigma}}}$

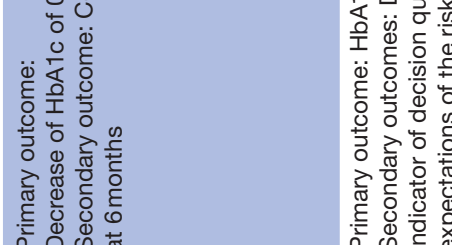

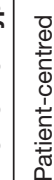
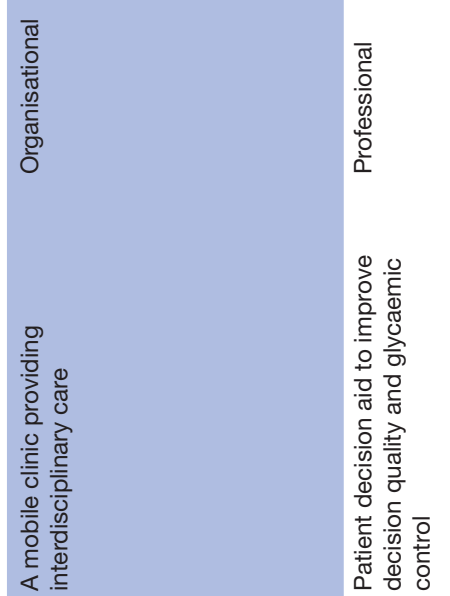

릉

ิㅡ

뜬

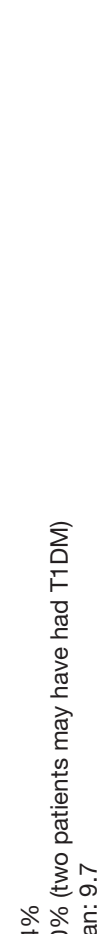

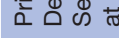

a o 5 这

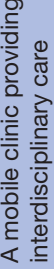

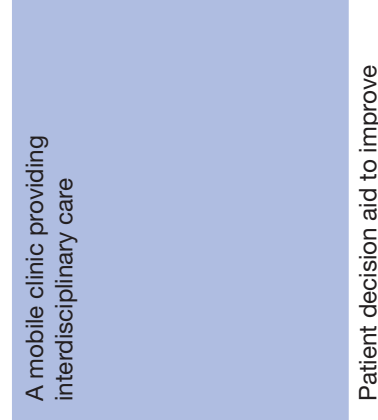




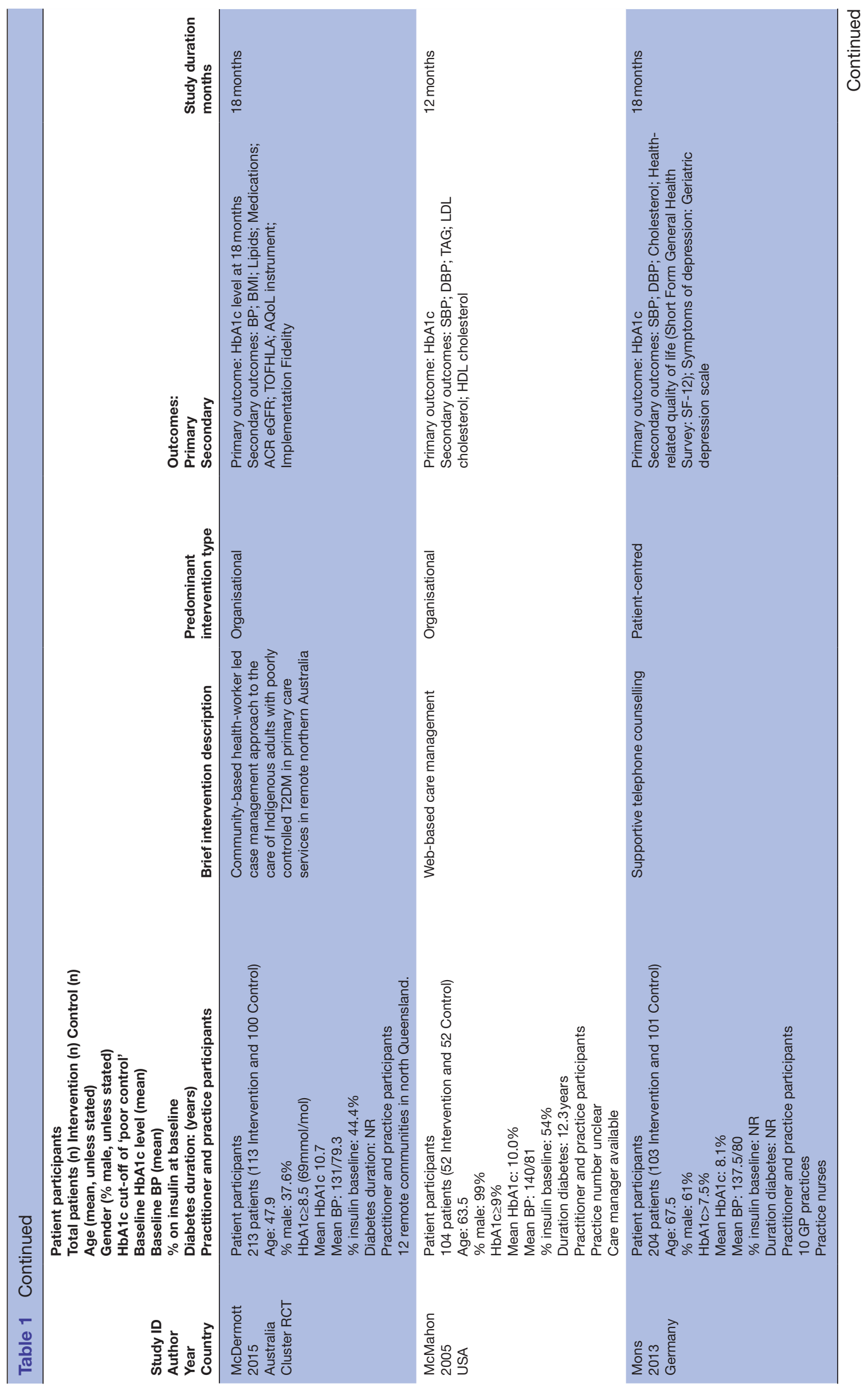

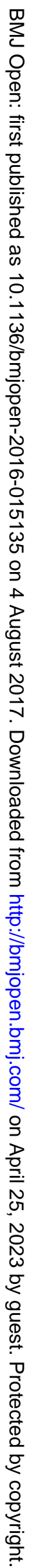



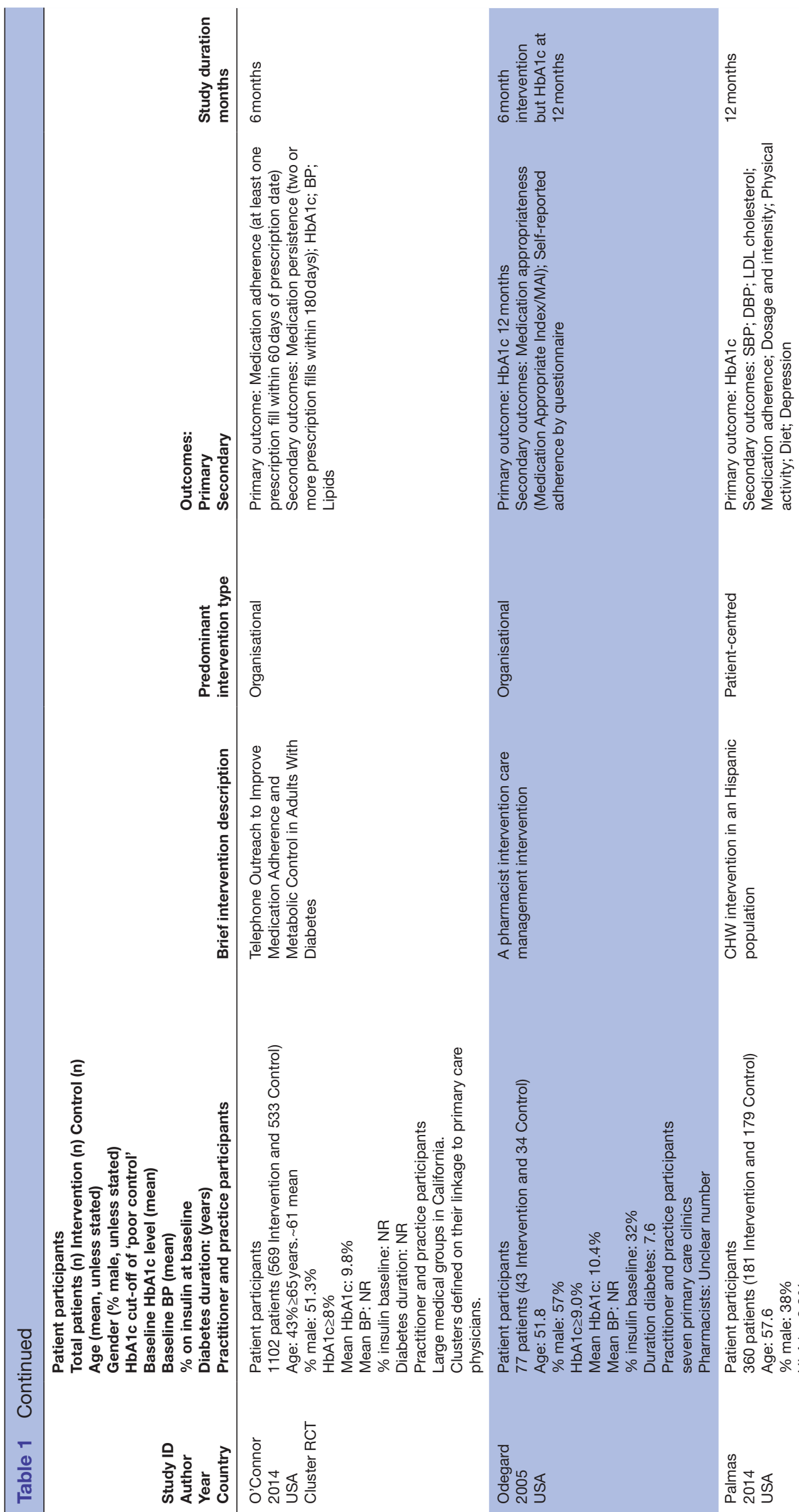

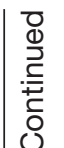

$\frac{\text { m }}{3}$

$\overrightarrow{\vec{F}}$

응

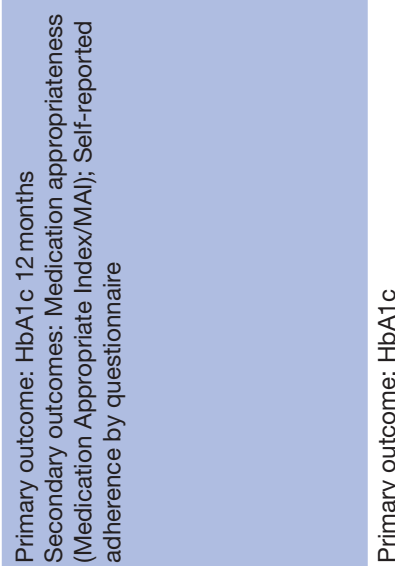

$\frac{\bar{\sigma}}{\overline{\frac{\pi}{\sigma}}}$

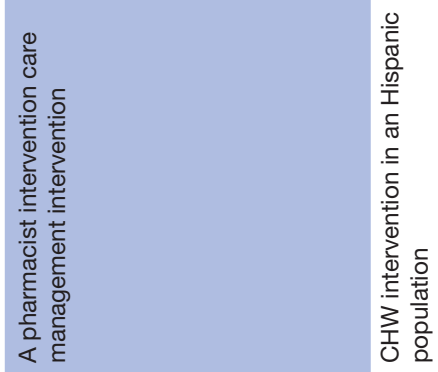

10 

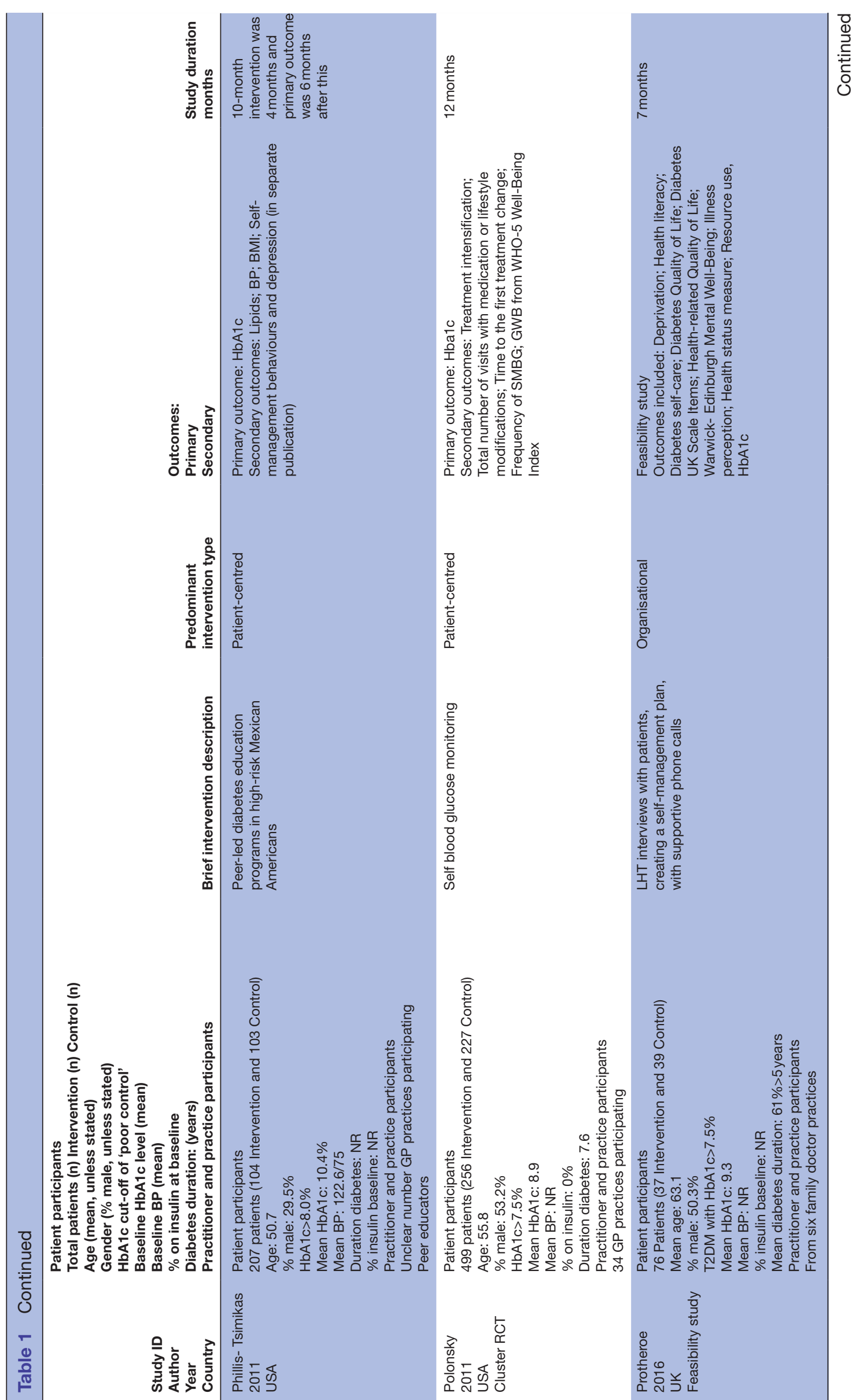

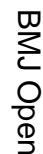

등 뭉

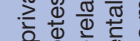

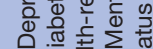

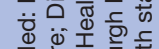

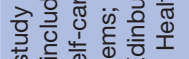

क.

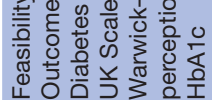

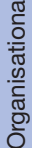
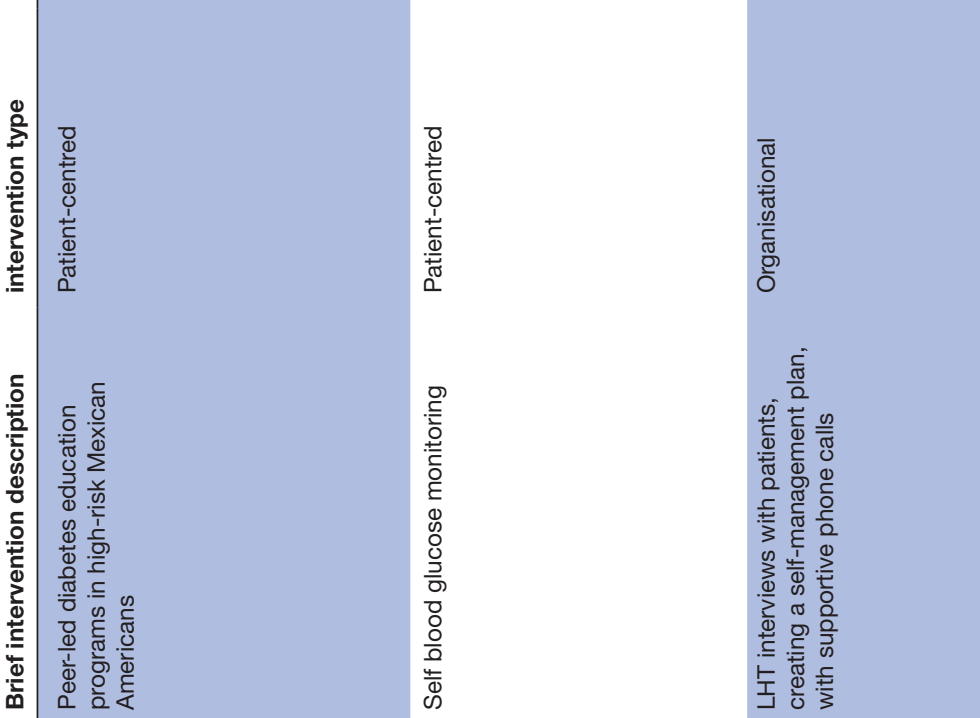

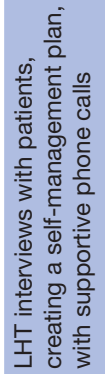

$\underline{\underline{a}}$

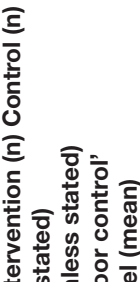

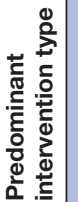

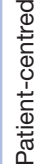

¿

के

옹
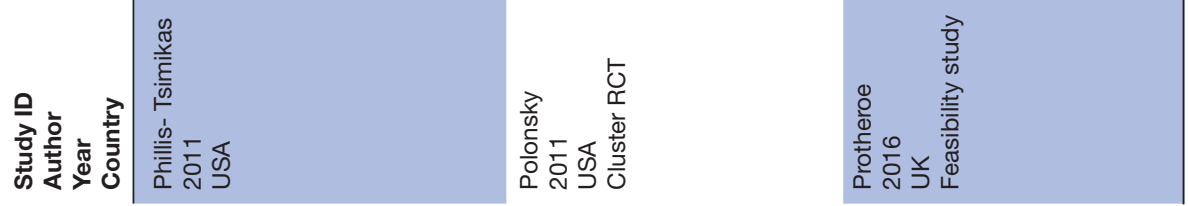


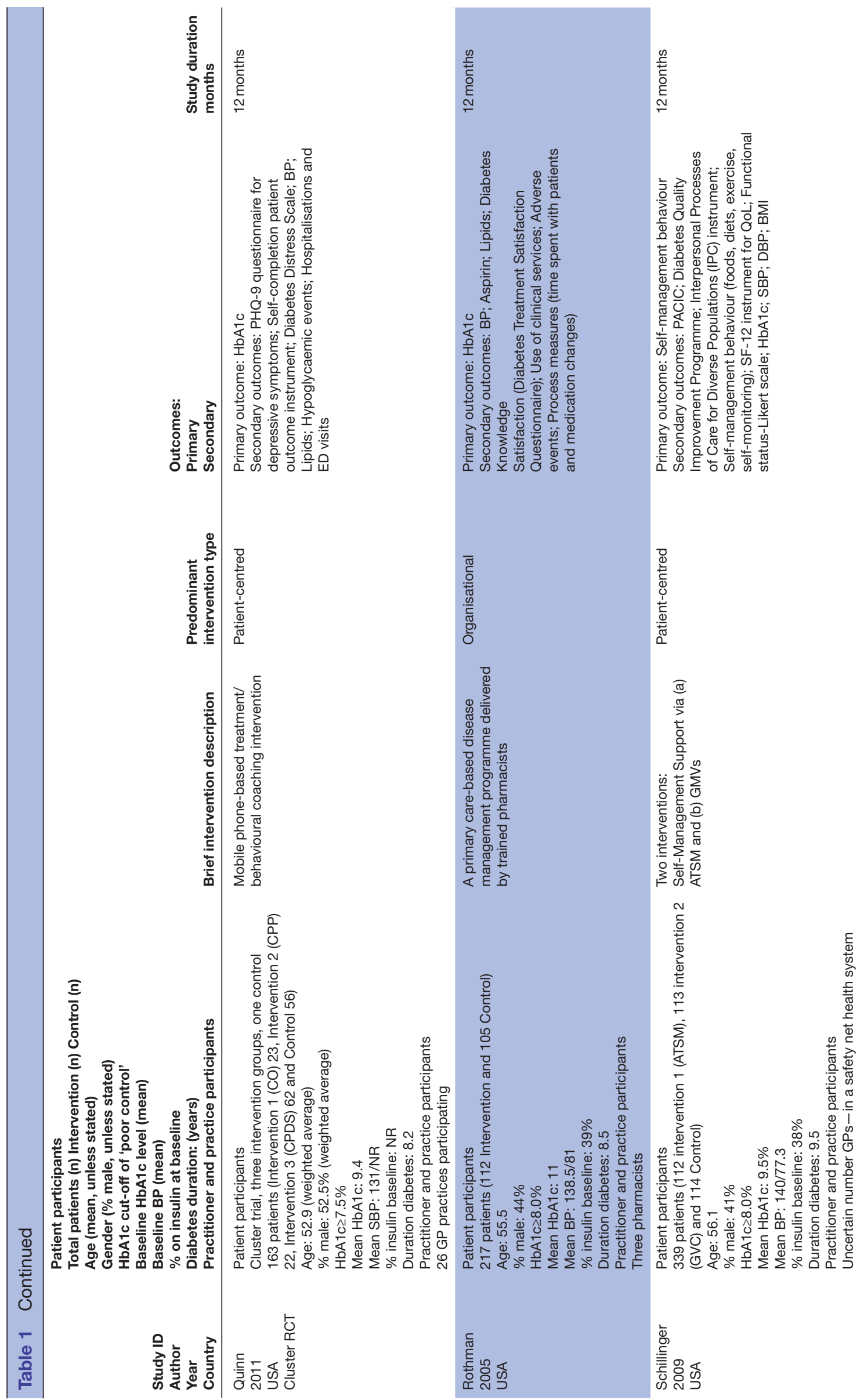

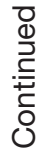



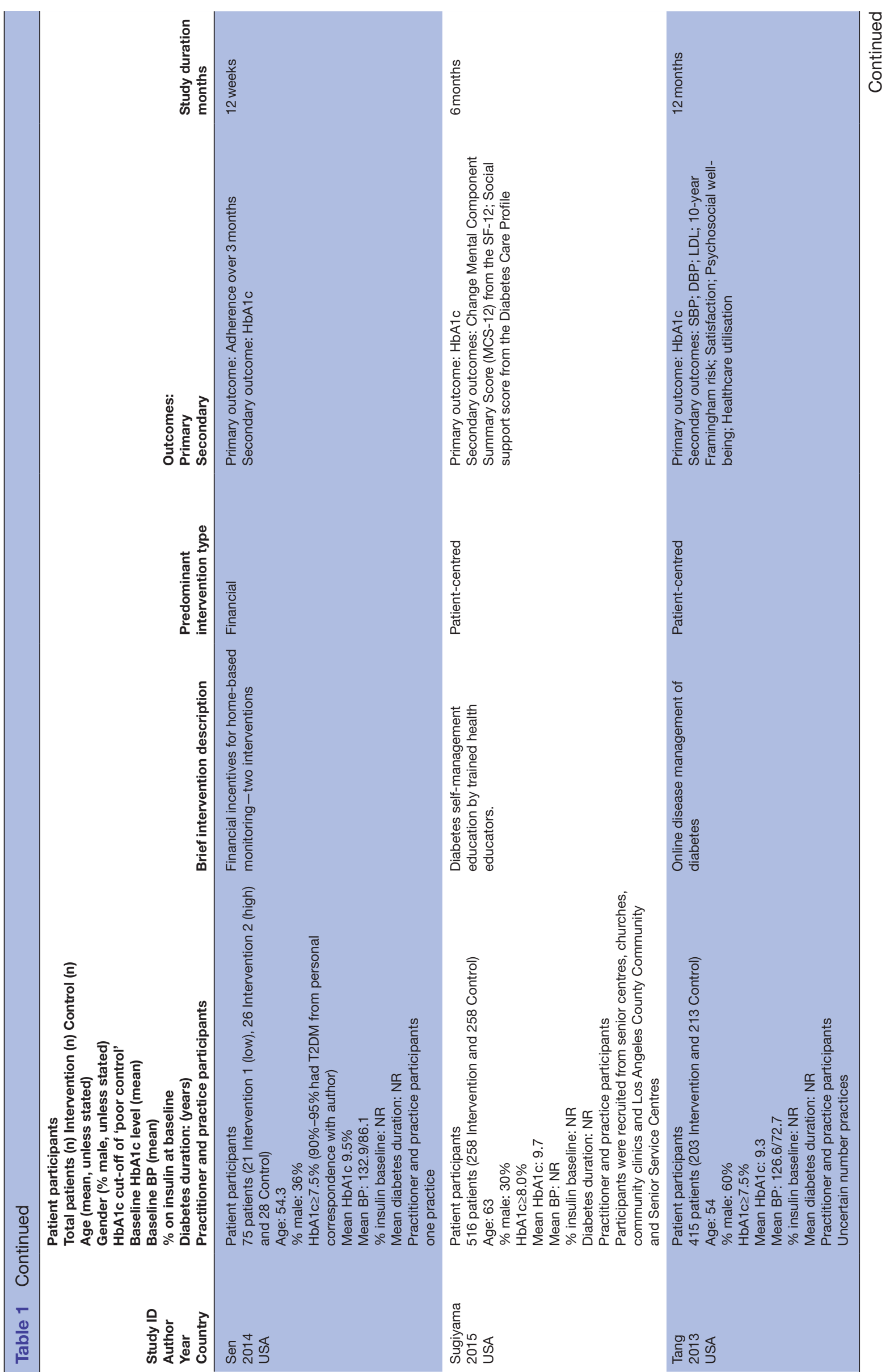

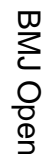

列

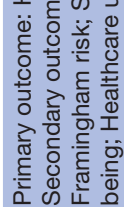

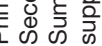

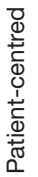

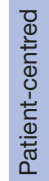
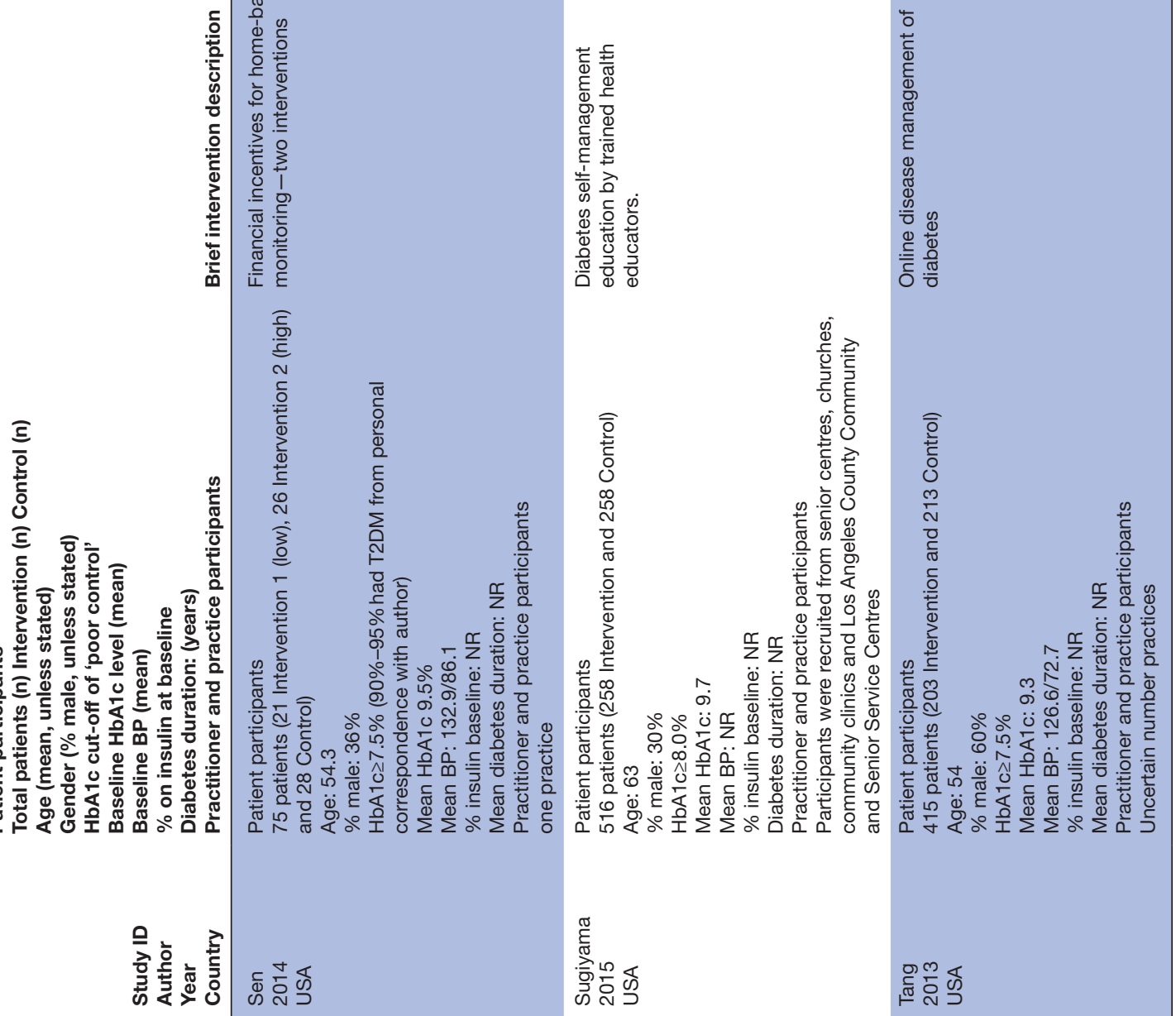


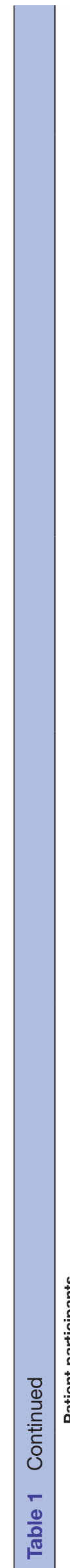

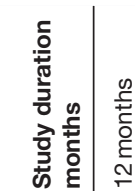

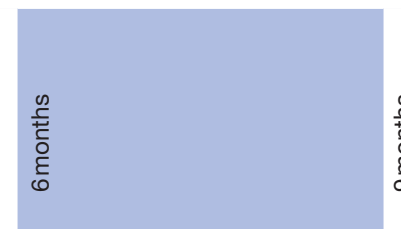

$\sum_{00}^{0}$

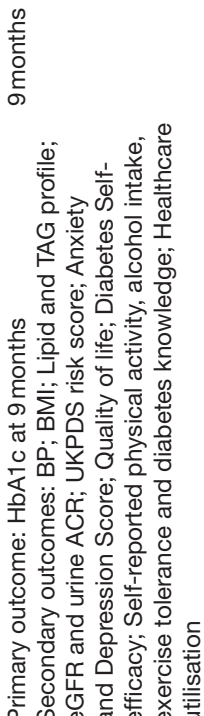

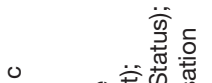

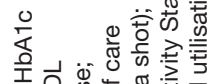

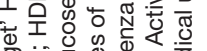

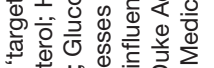

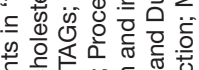

离

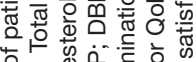

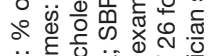

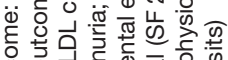

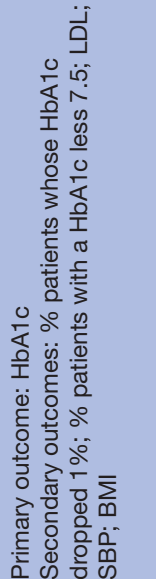

包 专

응

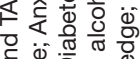

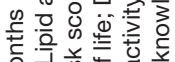

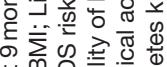

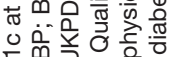

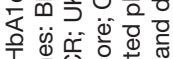

i.

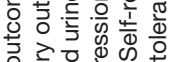

文䨌

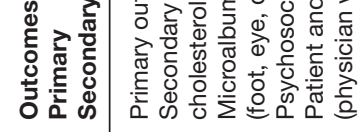

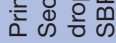

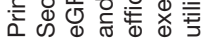

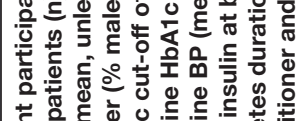

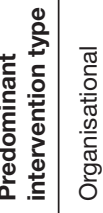

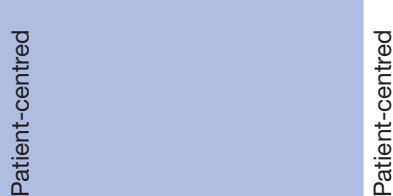

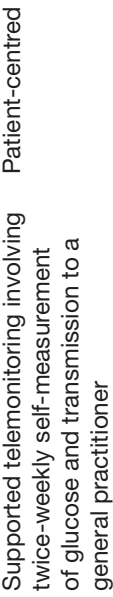

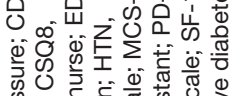

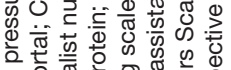

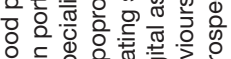

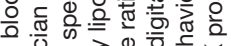

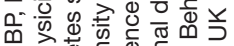

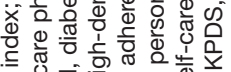

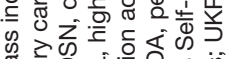

胥

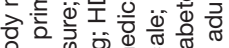

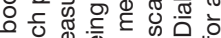

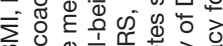

品 0

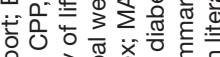

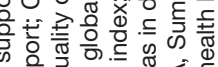

क 응

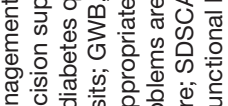

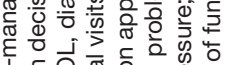

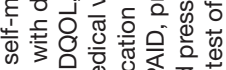

䉼

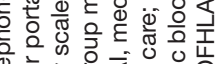

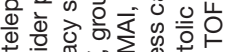

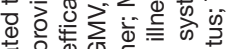

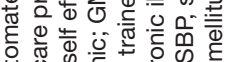

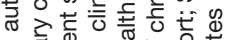

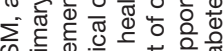

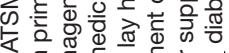

的宁

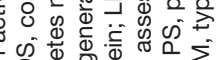

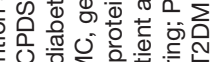

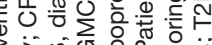

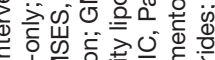

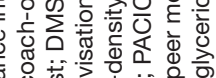

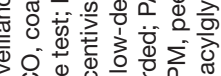

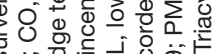

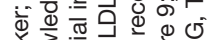

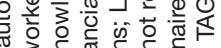

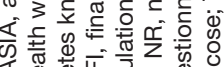

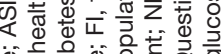

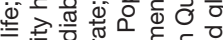

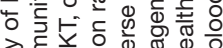

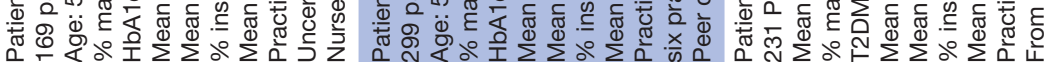

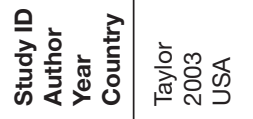

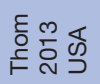

ำ 
mobile phone-based, ${ }^{32} 365261681$ video-based, ${ }^{51} 5$ peer${\text { support-based, }{ }^{30} 384449653 \text { self-monitoring-based }}^{37} 5064$ and 2 -culturally supportive self-management interventions. ${ }^{39}{ }^{45}$ The 20 organisational interventions included 5 pharmacist interventions performing case management, ${ }^{3540474857} 6$ nurse case management interventions, ${ }^{23} 31465355603$ web-based/telemedicine/telephone case management interventions, ${ }^{24} 5963$ new-clinic-based interventions, ${ }^{43} 54661$ community health-worker intervention, ${ }^{62} 1$ psychological intervention ${ }^{22}$ and 1 lay health worker intervention. ${ }^{67}$ Eight interventions had an mHealth or telehealth component. ${ }^{33} 36455256596568$ More detailed descriptions of the interventions are outlined in online supplementary appendix 3 .

\section{Risk of bias}

All 42 studies were RCTs, with six being cluster RCTs. Overall, 25 studies were classified as having a predominant low-risk of bias $(59.5 \%)$, ${ }^{22-24} 32-3639414245465153-5558$ 59 62-66 68 13 studies had an unclear-risk (31\%) $)^{30} 3137384044$ 47495657606167 and 4 RCTs were classified as having a highrisk of bias $(9.5 \%)^{43} 485052$ (see online supplementary appendix 4). Blinding of outcome assessment was classified as low-risk in all studies. Attrition bias was evident in seven studies. Online supplementary appendix 5 outlines the summary judgements for both overall risk of bias and predominant intervention type, which were used in the meta-regression analysis.

There was no evidence of publication bias in the studies included in the HbA1c $(\mathrm{p}=0.37)$ or SBP analysis $(\mathrm{p}=0.54)$. However, there was some evidence of publication bias in the studies included in the DBP analysis $(\mathrm{p}<0.01)$ (see online supplementary appendixes $6(\mathrm{a})$ and $6(\mathrm{~b}))$.

\section{Primary outcomes}

HbA1c

Overall 40 of the 42 studies were included in a meta-analysis, which found a MD in $\mathrm{HbA} 1 \mathrm{c}$ of $-3.7 \mathrm{mmol} / \mathrm{mol}$ $(-0.34 \%$; $95 \%$ CI $-0.46 \%$ to $-0.22 \%)$ favouring intervention groups, but with statistical heterogeneity $\left(\mathrm{I}^{2}=69 \%\right)$. Figure 2A outlines the overall effect of interventions on HbAlc, across EPOC categories.

Subgroup analyses were performed based on the predominant intervention type (figure 2A), the baseline HbA1c level (figure 2B), study duration (figure 2C) and study quality (figure 2D).

These analyses suggested that organisational interventions (MD in HbAlc of $-5.2 \mathrm{mmol} / \mathrm{mol}(-0.42 \%$; $95 \%$ CI $-0.66 \%$ to $-0.18 \%$; $\left.\mathrm{I}^{2}=79 \%\right)$ had better improvements in HbAlc than patient-centred interventions $(-0.30 \%$; $95 \%$ CI $-0.43 \%$ to $\left.-0.18 \% ; \mathrm{I}^{2}=48 \%\right) \quad(\mathrm{p}=0.05)$. Similarly interventions performed when the baseline population-HbAlc was over $80 \mathrm{mmol} / \mathrm{mol}(9.5 \%)$ (MD in $\mathrm{HbAlc}$ of $-6.3 \mathrm{mmol} / \mathrm{mol}(-0.58 \% ; 95 \%$ CI $-0.81 \%$ to $-0.35 \%$; $\left.\mathrm{I}^{2}=75 \%\right)$ had better improvements in HbAlc than populations with a baseline-HbAlc $<9.5 \%(-0.17 \% \%$; 95\% CI $-0.29 \%$ to $\left.-0.05 \% \% ; \mathrm{I}^{2}=51 \%\right) \quad(\mathrm{p}=0.002)$. Study duration did not appear to affect HbA1c (figure 2C). Lastly, studies with a low-risk of bias (MD in $\mathrm{HbA} 1 \mathrm{c}$ was $-2.8 \mathrm{mmol} / \mathrm{mol}$ $\left(-0.26 \% ; 95 \%\right.$ CI $-0.39 \%$ to $\left.-0.13 \% ; \mathrm{I}^{2}=59 \%\right)$ appeared to have a smaller reduction in HbAlc compared with unclear $\left(-0.49 \% \% ; 95 \% \mathrm{CI}-0.84 \% \%\right.$ to $\left.-0.15 \% ; \mathrm{I}^{2}=81 \%\right)$ and high-risk studies $(-0.41 \% ; 95 \% \mathrm{CI}-0.74 \%$ to $-0.09 \%$; $\mathrm{I}^{2}=61 \%$ ), but there was no evidence of a statistically significant difference $(p=0.35)$. Though not considered in our original protocol, subgroup analysis did not highlight additional benefit from those interventions (included in both organisational and patient-centred intervention types), which had a telemedicine or mHealth component (see online supplementary appendix 7)..$^{33} 36455256596568$

As the overall results showed statistical heterogeneity, meta-regression analysis was also conducted to explore the components of this heterogeneity. As with the meta-analyses, higher baseline HbAlc was associated with a greater reduction in HbA1c ( $\beta$-Coefficient: -0.27 ; $95 \%$ CI -0.41 to -0.13 ; $\mathrm{p}<0.001)$. The predominant-intervention type, risk of bias and study-duration were not associated with improved glycaemic control.

\section{Blood pressure}

Overall there was small improvement in SBP in the 26 interventions included in the meta-analysis, (MD SBP: $-1.13 \mathrm{~mm} \mathrm{Hg}(95 \% \mathrm{CI}-2.19$ to -0.08$)$ ) with moderate heterogeneity $\left(\mathrm{I}^{2}=47 \%\right)$ (see online supplementary appendix 8). $.^{22-24} 30-36383941454648-515458-6062656668$ DBP improved modestly in the 22 studies included in the meta-analysis (MD DBP: $-1.37 \mathrm{~mm} \mathrm{Hg}(95 \%$ CI -2.25 to -0.50$))$ with moderate heterogeneity $\left(\mathrm{I}^{2}=44 \%\right)$ (see online supplementary appendix 9). ${ }^{22-24} 313234-363839$ 41454648495154585962656668

In the subgroup analysis, organisational interventions appeared to improve SBP modestly (MD SBP: $-2.69 \mathrm{~mm} \mathrm{Hg} ; 95 \%$ CI -5.11 to $-0.26 ; \mathrm{I}^{2}=57 \%$ ) compared with patient-centred interventions (MD SBP: $-0.52 \mathrm{~mm} \mathrm{Hg} ; 95 \% \mathrm{CI}-1.41$ to $0.38 ; \mathrm{I}^{2}=20 \%$ ) which showed no statistically significant improvement (see online supplementary appendix 8). However, there was no evidence of a statistically significant difference between intervention types. Similarly with DBP, organisational interventions appeared to improve DBP modestly (MD DBP: $-2.87 \mathrm{~mm} \mathrm{Hg}$; $95 \%$ CI -4.29 to -1.45 ; $\left.\mathrm{I}^{2}=30 \%\right)$ compared with patient-centred interventions (MD DBP: $-1.37 \mathrm{~mm} \mathrm{Hg}$; $95 \% \mathrm{CI}-1.42$ to $0.2 ; \mathrm{I}^{2}=30 \%$ ) (see online supplementary appendix 9) and there was evidence of a statistically significant difference $(\mathrm{p}=0.007)$. Meta-regression analysis was not conducted for SBP or DBP, as significant heterogeneity was not present on the overall effect sizes.

Lipids

Twenty of the 42 studies reported total cholesterol, low-density lipoprotein cholesterol, high-density lipoprotein cholesterol or triacylglicerides. ${ }^{23} 2430-323536$ 38394145464851565862656668 Statistically significant improvements in lipids were only demonstrated in 4 of these 20 studies. ${ }^{32} 324548$ Baseline lipid levels were generally not reported. Eleven of the 20 studies reported data relating to total cholesterol. Meta-analysis was undertaken on 


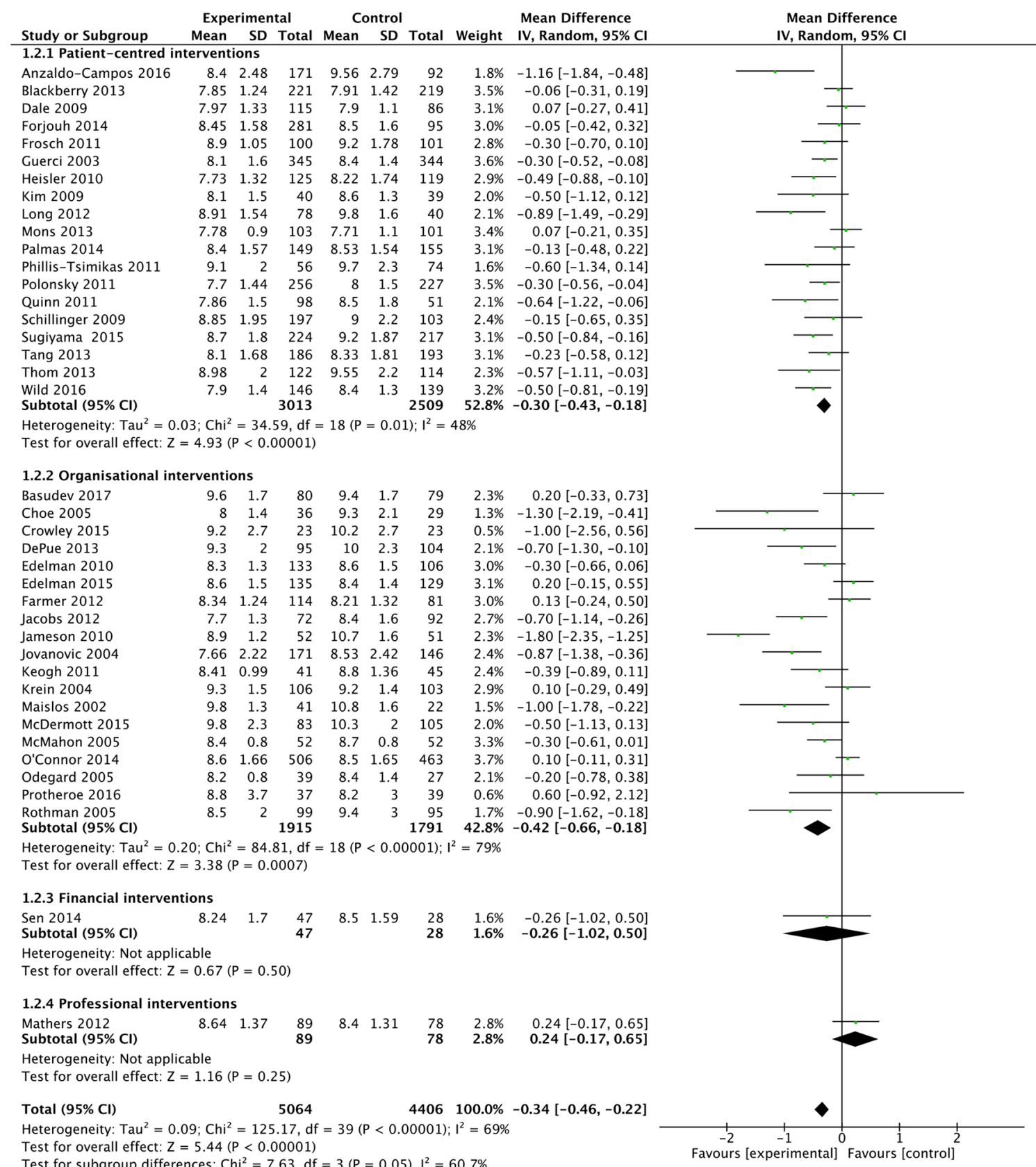

Figure 2A Effects of interventions on $\mathrm{HbA1c}$, with intervention-type subgroups.

these studies, which indicated a modest improvement in total cholesterol, favouring intervention groups (MD total cholesterol $-4.29 \mathrm{mg} / \mathrm{dL}(95 \% \mathrm{CI}-7.68$ to -0.89$)$; $\mathrm{I}^{2}=0 \%$ ) (see online supplementary appendix 10)..$^{353638}$ 4145465862656668

\section{Secondary outcomes}

All but 1 of the 42 included studies reported at least one of the eligible secondary outcomes (see online supplementary appendix 11). Overall, interventions had very limited effect on secondary outcomes. Twenty-six studies reported other physical outcomes (eg, body mass index (BMI) and estimated glomerular filtration rate). Of the 15 studies that reported on weight or BMI, only one showed significant improvement. ${ }^{56}$ Ten studies reported mental health outcomes 3638414558964 with two showing a significant improvement in the Change Mental Component Summary Score and the Short Form-12 Mental Health Score. ${ }^{64}{ }^{67}$ Twenty-eight studies reported PROMs, with 11 showing an improvement with the intervention. Ten studies reported medication adherence outcomes, with two showing improvement. Eighteen studies reported utilisation outcomes, with four improving processes of care.

\section{DISCUSSION}

\section{Statement of principle findings}

Healthcare interventions have positive, although modest, effects on HbA1c in poorly controlled T2DM. Interventions targeting those with a higher baseline HbAlc 


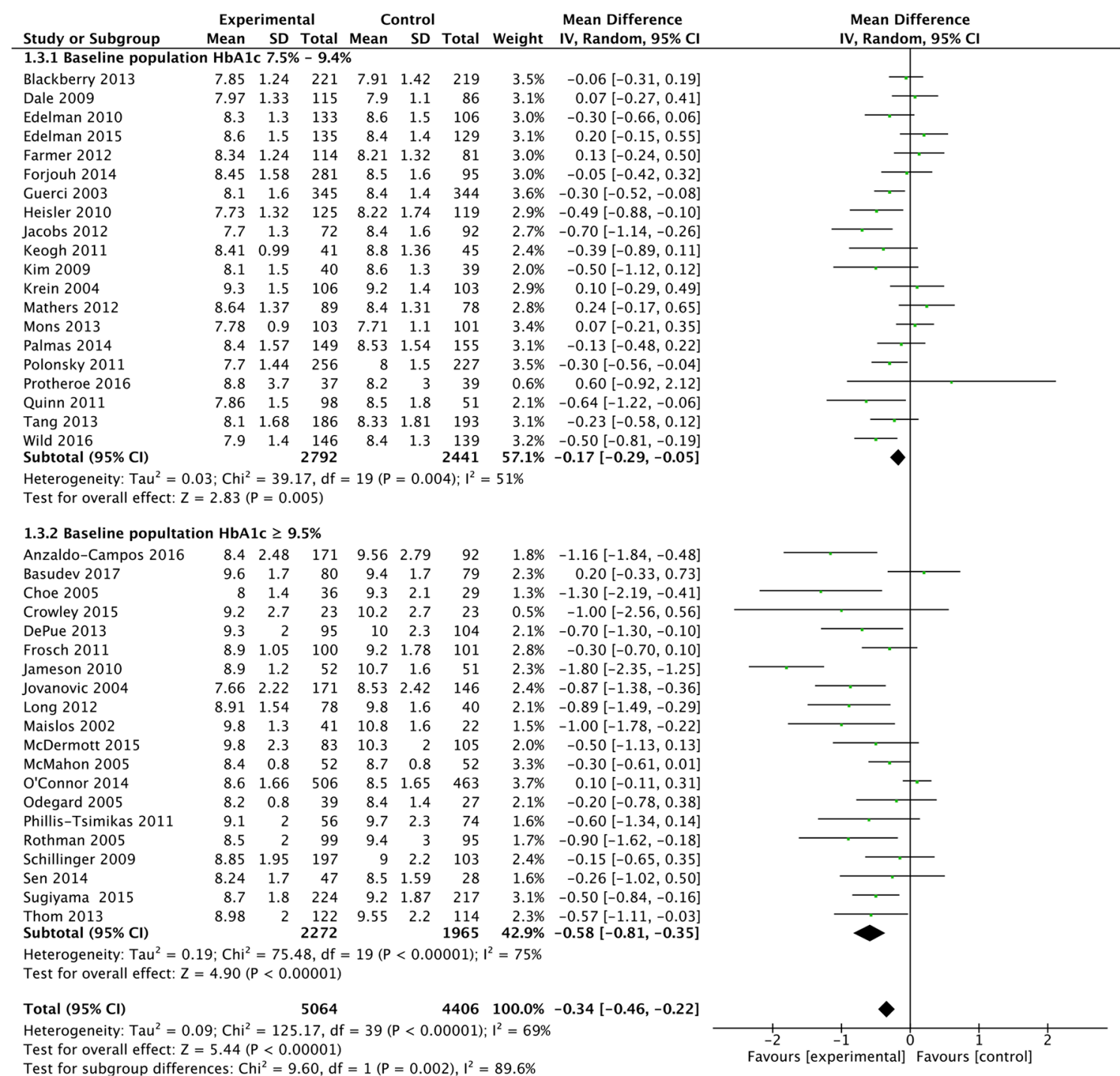

Figure 2B Effects of interventions on $\mathrm{HbA} 1 \mathrm{c}$, with baseline $\mathrm{HbA} 1 \mathrm{c}$ subgroup.

$(\geq 80 \mathrm{mmol} / \mathrm{mol}(9.5 \%))$ show the greatest effects. There was also evidence of a modest impact on both blood pressure and lipids, though baseline control of these risk factors was generally good. Generally, little effect on secondary outcomes was found. Our results suggest that a targeted approach to T2DM management, focussing on individuals with very poor glycaemic control, may represent a prudent strategy for future management.

\section{Strengths and weaknesses of the study}

The methodology of our systematic review addresses key credibility issues. ${ }^{69} 70$ The research question was sensible, our search of the literature was exhaustive and our results are outlined clearly for primary and secondary outcomes. The effect of baseline HbAlc was consistent across studies, biologically plausible and was an a priori hypothesis. ${ }^{70}$

We performed meta-regression to explore the heterogeneity, which also confirmed the increased effectiveness of interventions on those with $\mathrm{HbAlc} \geq 80 \mathrm{mmol} / \mathrm{mol}$ $(9.5 \%)$. However, a major limitation is that meta-regression is usually underpowered to detect anything but very large associations. Meta-regression considers the interactions between trial level covariates and the treatment effect, but it inherits difficulties of interpretation attached to non-randomised studies, as it is not possible to randomise patients to one covariate value or another, so causality cannot be attached its findings. ${ }^{71}$ Though we do not believe the subgroup findings occurred by chance, there remained high heterogeneity and we explored between-study comparisons rather than within-study comparisons. ${ }^{70}$ There was some evidence of publication bias in the DBP analysis, but this was not present for the 22 studies reporting SBP. It should also be noted that the power of Egger's test is low when the number of studies is small and should only be used if the analysis includes a range of study sizes.

This study will inform researchers regarding the range of interventions that have been deployed to target patients with poorly controlled T2DM. There is no specific definition for 'poor control' of T2DM in the literature, but by including all studies that had patients with a $\mathrm{HbAlc}>59 \mathrm{mmol} / \mathrm{mol}(7.5 \%)$, we captured the full range of poor glycaemic control. Studies examining poor 


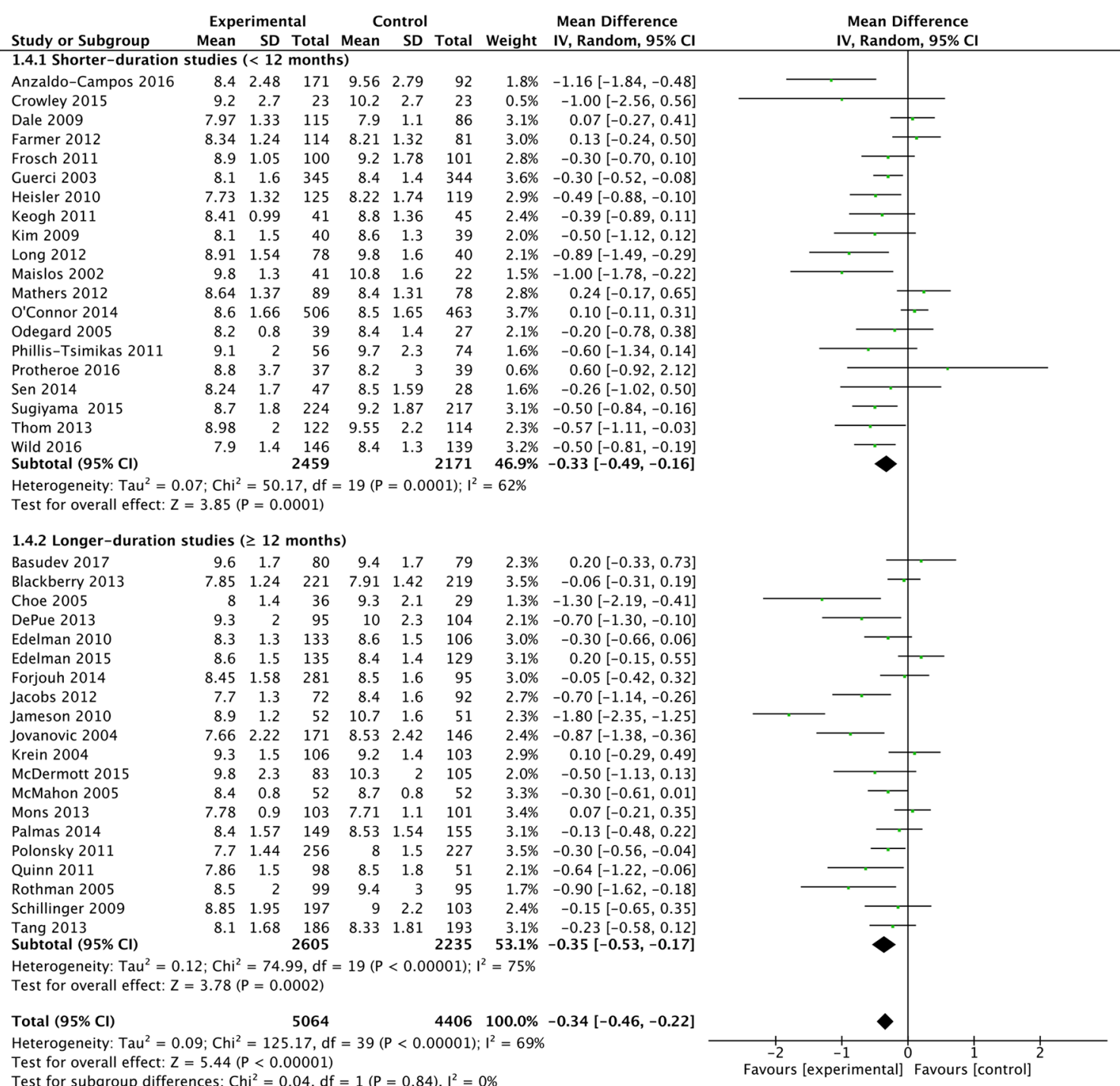

Figure $2 \mathrm{C}$ Effects of interventions on $\mathrm{HbA} 1 \mathrm{c}$, with baseline study duration subgroups.

control of HbAlc possess a risk of regression towards the mean. However, all included studies were RCTs with control groups, which should have accounted for this. Targeted interventions in poorly controlled T2DM need to be distinguished from interventions, which are designed to intensively reduce $\mathrm{HbA1c}$ in all patients. Though persons with very poor glycaemic control are also at risk of the adverse effects of hypoglycaemic agents, targeting this population is more likely to reach the right balance of reducing harms of overtreatment and maximising potential benefits. ${ }^{18}$ The relative importance of targeting glycaemic or cardiovascular risk has been debated in the literature. ${ }^{17}$ We did not account for medication use in the studies, but given that all included studies were RCTs, which would balance out delivery of medications, we think that differences relating to underlying medication usage relate to how different interventions types promote the intensification of medications.

\section{Comparison with other studies}

The existing literature examining healthcare interventions to improve glycaemic control has focused on a range of approaches. There have been systematic reviews of interventions including QI initiatives, education, self-management support, case-management, adherence to medication and professional interventions, though as outlined previously, most have not specifically targeted patients with poor glycaemic control. ${ }^{81011}$

A synthesis of 27 systematic reviews and 347 RCTs identified the cost-effectiveness of self-management interventions in T2DM in all patients with T2DM. ${ }^{72}$ This overview included studies that targeted all patients with T2DM and found very good evidence that education improves blood glucose control in patients with T2DM in the short term (less than 12 months) and that behavioural and psychological interventions are associated with modest improvements in blood glucose control (HbA1C). ${ }^{72} 73$ A review of computer-based diabetes self-management interventions to manage T2DM reported a small beneficial effect on blood glucose control (MD of $-0.2 \%){ }^{74}$ Another recent systematic review of 118 self-management interventions found improvements in HbAlc in $62 \%$ of studies. The overall mean effect was to reduce HbAlc 


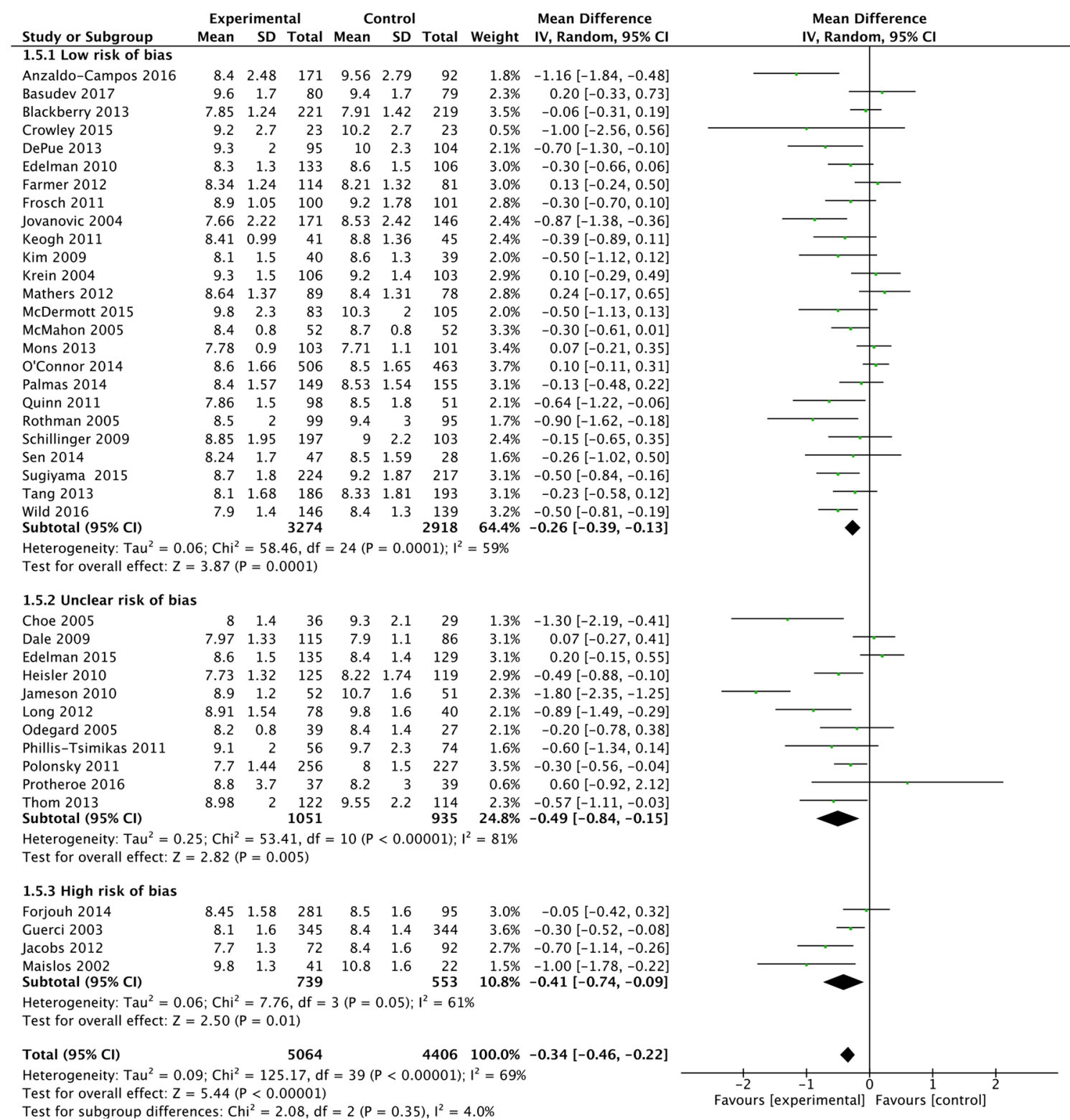

Figure 2D Effects of interventions on $\mathrm{HbA} 1 \mathrm{c}$, with baseline study quality subgroup.

by $-0.57 \%$, although patients with persistently elevated HbA1c over 9 had greater improvements. ${ }^{75}$ In our review, patient-orientated interventions, such as self-monitoring of blood glucose and self-management interventions, seemed to be less effective than organisational interventions.

Case management by nurses and other professionals and case management in socially disadvantaged have been shown to be beneficial when targeted at all patients with T2DM and our review supports this conclusion for poorly controlled populations. ${ }^{5}$ 76-78 Pharmacist-based interventions have been studied, mainly in outpatient settings or in US primary care and have been found to be effective and cost-effective. ${ }^{79} 80$ The five pharmacist interventions in our review, targeting patients with poorly controlled T2DM, showed mixed results, but overall had predominantly positive effects on HbAlc.
Attention to, and reporting of, intensification of antidiabetic medications and patient's adherence to treatment regimens are needed to achieve optimal glycaemic control. ${ }^{81}{ }^{82}$ Evidence regarding adherence in T2DM is mixed. A previous systematic review of 21 studies that included 14 RCTs to enhance T2DM treatment adherence in community and hospital settings found that few studies measured or assessed adherence and that interventions to improve adherence did not show benefits or harms. ${ }^{83}$ A review by Farmer et al found limited evidence of effect for interventions promoting the monitoring of medication use and brief messaging to support medication adherence in patients with T2DM, though the included studies did not specifically target patients with poorly controlled diabetes. ${ }^{84}$ Only 10 of the 42 included studies in our review looked at adherence to medications as an outcome and only 2 of these 9 studies had a statistically 
significant effect on adherence. ${ }^{492}$ The baseline level of adherence varied considerably and studies used different scale ranges.

Our review identified only one professional-based interventions in poorly controlled T2DM, through a physician decision aid. ${ }^{42}$ Two systematic reviews have examined the impact of clinical decision support systems (CDSS) on the management of T2DM in primary care, between them looking at 28 trials, with varying results but none of these CDSS interventions were designed to promote intensification of prescribing in persons with poor glycaemic control. ${ }^{85} 86$

\section{Future research}

There is a need for further research examining professional-based interventions in poorly controlled T2DM, such as CDSS, which promote intensification of medications. ${ }^{81}$ Studies from jurisdictions outside North America on poorly controlled populations would also be welcome. An individual patient data meta-analysis would answer further questions not possible in this review and future research should attempt to obtain individual-level patient data. It is likely that most successful interventions have their impact as a result of intensification of medicines and/or improving adherence to medicines. ${ }^{81}$ As adherence was not measured in most of the studies and intensification poorly documented, it is important that future interventions report on these findings. Furthermore organisational interventions could incur significant costs to a health system, so cost-effectiveness analyses on future interventions should be undertaken to ensure the modest improvements in $\mathrm{HbAlc}$ are beneficial for the health systems.

In conclusion, clinicians and policy makers, when considering organisation of care for T2DM, should focus their effects on those patients with very poor glycaemic control $(\geq 80 \mathrm{mmol} / \mathrm{mol}(9.5 \%))$. Prioritising interventions that emphasise structured organisation of care, which can include intensification and adherence to medications, also seem more likely to deliver optimal results in terms of glycaemic control for T2DM patients.

Contributors All authors contributed to the drafting of the paper. MEM and RG independently assessed studies for eligibility, extracted data and assessed study quality. Decisions or disagreements were brought to SMS. SMS, TF and FB provided methodological and statistical support to the paper.

Funding This work was supported by the HRB Centre for Primary Care Research (Research Grant: HRC-2014-1), a publicly funded body. Four of the six study authors are employed by this agency.

Competing interests None declared.

Provenance and peer review Not commissioned; externally peer reviewed.

Data sharing statement All collected data has been supplied as Supplementary Files. Please contact the corresponding author (MEM) if there are queries regarding this data.

Open Access This is an Open Access article distributed in accordance with the Creative Commons Attribution Non Commercial (CC BY-NC 4.0) license, which permits others to distribute, remix, adapt, build upon this work non-commercially, and license their derivative works on different terms, provided the original work is properly cited and the use is non-commercial. See: http://creativecommons.org/ licenses/by-nc/4.0/ (c) Article author(s) (or their employer(s) unless otherwise stated in the text of the article) 2017. All rights reserved. No commercial use is permitted unless otherwise expressly granted.

\section{REFERENCES}

1. Wild S, Roglic G, Green A, et al. Global prevalence of diabetes: estimates for the year 2000 and projections for 2030. Diabetes Care 2004;27:1047-53.

2. Spann SJ, Nutting PA, Galliher JM, et al. Management of type 2 diabetes in the primary care setting: a practice-based research network study. Ann Fam Med 2006;4:23-31.

3. Campbell DJ, McGrady M, Prior DL, et al. Most individuals with treated blood pressures above target receive only one or two antihypertensive drug classes. Intern Med J 2013;43:137-43

4. Stratton IM, Adler Al, Neil HA, et al. Association of glycaemia with macrovascular and microvascular complications of type 2 diabetes (UKPDS 35): prospective observational study. BMJ 2000;321:405-12.

5. Stellefson M, Dipnarine K, Stopka C. The chronic care model and diabetes management in US primary care settings: a systematic review. Prev Chronic Dis 2013;10:E26.

6. Mays N. Reducing unwarranted variations in healthcare in the English NHS. BMJ 2011;342:d1849.

7. Simmons RK, Carlsen AH, Griffin SJ, et al. Variation in prescribing of lipid-lowering medication in primary care is associated with incidence of cardiovascular disease and all-cause mortality in people with screen-detected diabetes: findings from the ADDITIONDenmark trial. Diabet Med 2014;31:1577-85.

8. Seitz P, Rosemann T, Gensichen J, et al. Interventions in primary care to improve cardiovascular risk factors and glycated haemoglobin $(\mathrm{HbA1c})$ levels in patients with diabetes: a systematic review. Diabetes Obes Metab 2011;13:479-89.

9. Renders CM, Valk GD, Griffin SJ, et al. Interventions to improve the management of diabetes in primary care, outpatient, and community settings: a systematic review. Diabetes Care 2001;24:1821-33.

10. Seidu S, Walker NS, Bodicoat DH, et al. A systematic review of interventions targeting primary care or community based professionals on cardio-metabolic risk factor control in people with diabetes. Diabetes Res Clin Pract 2016;113:1-13.

11. Tricco AC, Ivers NM, Grimshaw JM, et al. Effectiveness of quality improvement strategies on the management of diabetes: a systematic review and meta-analysis. Lancet 2012;379:2252-61.

12. Patel A, MacMahon S, Chalmers J, et al. Intensive blood glucose control and vascular outcomes in patients with type 2 diabetes. $N$ Engl J Med 2008;358:2560-72.

13. Gerstein HC, Miller ME, Byington RP, et al. Effects of intensive glucose lowering in type 2 diabetes. $N$ Engl $\mathrm{J}$ Med 2008;358:2545-59.

14. Duckworth W, Abraira C, Moritz T, et al. Glucose control and vascular complications in veterans with type 2 diabetes. $N$ Engl J Med 2009;360:129-39.

15. Turnbull FM, Abraira C, Anderson RJ, et al. Intensive glucose control and macrovascular outcomes in type 2 diabetes. Diabetologia 2009;52:2288-98.

16. Skyler JS, Bergenstal R, Bonow RO, et al. Intensive glycemic control and the prevention of cardiovascular events: implications of the ACCORD, ADVANCE, and VA Diabetes Trials: a position statement of the american Diabetes Association and a Scientific Statement of the American College of Cardiology Foundation and the american Heart Association. J Am Coll Cardiol 2009;53:298-304.

17. Hayward RA, Reaven PD, Wiitala WL, et al. Follow-up of glycemic control and cardiovascular outcomes in type 2 diabetes. $N$ Engl J Med 2015;372:2197-206.

18. Hayward RA. Excessive testing of adults with type 2 diabetes. BMJ 2015;351:h6549.

19. Mossello E. Targeting vascular risk factors in older adults: from Polypill to Personalized Prevention. JAMA Intern Med 2015;175:1949-50.

20. Murphy M, Galvin R, Fahey T, et al. Effectiveness of interventions in primary care to improve glycated haemoglobin (HbA1c) and cardiovascular risk factor levels in patients with poorly-controlled type 2 diabetes mellitus: a systematic review. PROSPERO 2014. CRD42014014442.

21. Effective practice and Organisation of Care. EPOC Intervention types. Norwegian Knowledge Centre for the Health Services. 2015. 
accessed on 13 April 2016. https://epoc.cochrane.org/sites/epoc. cochrane.org/files/uploads/EPOC.

22. Keogh KM, Smith SM, White P, et al. Psychological family intervention for poorly controlled type 2 diabetes. Am J Manag Care 2011;17:105-13.

23. Krein SL, Klamerus ML, Vijan S, et al. Case management for patients with poorly controlled diabetes: a randomized trial. Am J Med 2004;116:732-9.

24. McMahon GT, Gomes HE, Hickson Hohne S, Hohne SH, et al. Webbased care management in patients with poorly controlled diabetes. Diabetes Care 2005;28:1624-9.

25. Vanselow NA. A New Definition of Primary Care. JAMA: The Journal of the American Medical Association 1995;273:192.

26. Effective practice and Organisation of Care (EPOC). Summary assessments of the risk of Bias. EPOC Resources for review authors Oslo: Norwegian Knowledge Centre for the Health Services 20132016 http://epoc.cochrane.org/sites/epoc.cochrane.org/files/ uploads $/ 16$.

27. StataCorp. Stata Statistical Software: release 13. College Station, TX: StataCorp, 2013. LP.

28. Higgins JP, Thompson SG. Quantifying heterogeneity in a metaanalysis. Stat Med 2002;21:1539-58.

29. Thompson SG, Higgins JP. How should meta-regression analyses be undertaken and interpreted? Stat Med 2002;21:1559-73.

30. Thom DH, Ghorob A, Hessler D, et al. Impact of peer health coaching on glycemic control in low-income patients with diabetes: a randomized controlled trial. Ann Fam Med 2013;11:137-44.

31. Taylor CB, Miller NH, Reilly KR, et al. Evaluation of a nursecare management system to improve outcomes in patients with complicated diabetes. Diabetes Care 2003;26:1058-63.

32. Tang PC, Overhage JM, Chan AS, et al. Online disease management of diabetes: engaging and motivating patients online with enhanced resources-diabetes (EMPOWER-D), a randomized controlled trial. $J$ Am Med Inform Assoc 2013;20:526-34.

33. Sen AP, Sewell TB, Riley EB, et al. Financial incentives for homebased health monitoring: a randomized controlled trial. J Gen Intern Med 2014;29:770-7.

34. Schillinger D, Handley M, Wang F, et al. Effects of self-management support on structure, process, and outcomes among vulnerable patients with diabetes: a three-arm practical clinical trial. Diabetes Care 2009;32:559-66.

35. Rothman RL, Malone R, Bryant B, et al. A randomized trial of a primary care-based disease management program to improve cardiovascular risk factors and glycated hemoglobin levels in patients with diabetes. Am J Med 2005;118:276-84.

36. Quinn CC, Shardell MD, Terrin ML, et al. Cluster-randomized trial of a mobile phone personalized behavioral intervention for blood glucose control. Diabetes Care 2011;34:1934-42.

37. Polonsky WH, Fisher L, Schikman $\mathrm{CH}$, et al. A structured selfmonitoring of blood glucose approach in type 2 diabetes encourages more frequent, intensive, and effective physician interventions: results from the STeP study. Diabetes Technol Ther 2011;13:797-802.

38. Philis-Tsimikas A, Fortmann A, Lleva-Ocana L, et al. Peer-led diabetes education programs in high-risk mexican Americans improve glycemic control compared with standard approaches: a project Dulce Promotora randomized trial. Diabetes Care 2011;34:1926-31.

39. Palmas W, Findley SE, Mejia M, et al. Results of the Northern Manhattan Diabetes community outreach project: a Randomized trial studying a community health worker intervention to improve diabetes care in Hispanic adults. Diabetes Care 2014;37:963-9.

40. Odegard PS, Goo A, Hummel J, et al. Caring for poorly controlled diabetes mellitus: a randomized pharmacist intervention. Ann Pharmacother 2005;39:433-40.

41. Mons U, Raum E, Krämer HU, et al. Effectiveness of a Supportive Telephone counseling intervention in type 2 Diabetes Patients: randomized Controlled Study. PLoS One 2013;8:e77954.

42. Mathers $\mathrm{N}, \mathrm{Ng}$ CJ, Campbell MJ, et al. Clinical effectiveness of a patient decision aid to improve decision quality and glycaemic control in people with diabetes making treatment choices: a cluster randomised controlled trial (PANDAs) in general practice. BMJ Open 2012;2:e001469.

43. Maislos M, Weisman D. Multidisciplinary approach to patients with poorly controlled type 2 diabetes mellitus: a prospective, randomized study. Acta Diabetol 2004:41:44-8.

44. Long JA, Jahnle EC, Richardson DM, et al. Peer mentoring and financial incentives to improve glucose control in african american veterans: a randomized trial. Ann Intern Med 2012;156:416-24.

45. Kim MT, Han HR, Song HJ, et al. A community-based, culturally tailored behavioral intervention for korean Americans with type 2 diabetes. Diabetes Educ 2009;35:986-94.
46. Jovanovic L. California Medi-Cal Type 2 Diabetes Study Group. Closing the gap: effect of diabetes case management on glycemic control among low-income ethnic minority populations: the California Medi-Cal type 2 diabetes study. Diabetes Care 2004;27:95-103.

47. Jameson JP, Baty PJ. Pharmacist collaborative management of poorly controlled diabetes mellitus: a randomized controlled trial. Am J Manag Care 2010;16:250-5.

48. Jacobs M, Sherry PS, Taylor LM, et al. Pharmacist Assisted Medication Program enhancing the regulation of Diabetes (PAMPERED) study. Journal of the American Pharmacists Association 2012;52:613-21.

49. Heisler M, Vijan S, Makki F, et al. Diabetes control with reciprocal peer support versus nurse care management: a randomized trial. Ann Intern Med 2010;153:507-15.

50. Guerci B, Drouin P, Grangé V, et al. Self-monitoring of blood glucose significantly improves metabolic control in patients with type 2 diabetes mellitus: the Auto-Surveillance intervention active (ASIA) study. Diabetes Metab 2003;29:587-94.

51. Frosch DL. Evaluation of a Behavior Support Intervention for Patients With Poorly Controlled Diabetes. Arch Intern Med 2011;171:2011-7.

52. Forjuoh SN, Bolin JN, Huber JC, et al. Behavioral and technological interventions targeting glycemic control in a racially/ethnically diverse population: a randomized controlled trial. BMC Public Health 2014;14:71.

53. Farmer A, Hardeman W, Hughes D, et al. An explanatory randomised controlled trial of a nurse-led, consultation-based intervention to support patients with adherence to taking glucose lowering medication for type 2 diabetes. BMC Fam Pract 2012;13:30.

54. Edelman D, Fredrickson SK, Melnyk SD, et al. Medical clinics versus usual care for patients with both diabetes and hypertension: a randomized trial. Ann Intern Med 2010;152:689-96.

55. DePue JD, Dunsiger S, Seiden AD, et al. Nurse-community health worker team improves diabetes care in American Samoa: results of a randomized Controlled trial. Diabetes Care 2013;36:1947-53.

56. Dale J, Caramlau I, Sturt J, et al. Telephone peer-delivered intervention for diabetes motivation and support: the telecare Exploratory RCT. Patient Educ Couns 2009;75:91-8.

57. Choe HM, Mitrovich S, Dubay D, et al. Proactive case management of high-risk patients with type 2 diabetes mellitus by a clinical pharmacist: a randomized controlled trial. Am J Manag Care 2005;11:253-60.

58. Blackberry ID, Furler JS, Best JD, et al. Effectiveness of general practice based, practice nurse led telephone coaching on glycaemic control of type 2 diabetes: the patient engagement and coaching for Health $(\mathrm{PEACH})$ pragmatic cluster randomised controlled trial. $B M J$ 2013;347:f5272.

59. Crowley MJ, Edelman D, McAndrew AT, et al. Effectiveness of a scalable telemedicine intervention for veterans with persistent poor diabetes control. Diabetes 2015;64:A80.

60. Edelman D, Dolor RJ, Coffman CJ, et al. Nurse-led behavioral management of diabetes and hypertension in community practices: a randomized trial. J Gen Intern Med 2015;30:626-33.

61. Capozza K, Woolsey S, Georgsson M, et al. Going mobile with diabetes support: a randomized study of a text message-based personalized behavioral intervention for type 2 diabetes self-care. Diabetes Spectr 2015;28:83-91.

62. McDermott RA, Schmidt B, Preece C, et al. Community health workers improve diabetes care in remote Australian Indigenous communities: results of a pragmatic cluster randomized controlled trial. BMC Health Serv Res 2015;15:68.

63. O'Connor PJ, Schmittdiel JA, Pathak RD, et al. Randomized trial of telephone outreach to improve medication adherence and metabolic control in adults with diabetes. Diabetes Care 2014;37:3317-24.

64. Sugiyama T, Steers WN, Wenger NS, et al. Effect of a communitybased diabetes self-management empowerment program on mental health-related quality of life: a causal mediation analysis from a randomized controlled trial. BMC Health Serv Res 2015;15:115

65. Anzaldo-Campos MC, Contreras S, Vargas-Ojeda A, et al. Dulce Wireless Tijuana: a Randomized Control Trial evaluating the impact of Project Dulce and Short-Term Mobile Technology on Glycemic Control in a Family Medicine Clinic in Northern Mexico. Diabetes Technol Ther 2016;18:240-51.

66. Basudev N, Crosby-Nwaobi R, Thomas S, et al. A prospective randomized controlled study of a virtual clinic integrating primary and specialist care for patients with type 2 diabetes mellitus. Diabet Med 2016;33:768-76.

67. Protheroe J, Rathod T, Bartlam B, et al. The feasibility of Health Trainer Improved Patient Self-Management in patients with Low Health literacy and poorly controlled Diabetes: a pilot Randomised Controlled Trial. J Diabetes Res 2016;2016:1-11. 
68. Wild SH, Hanley J, Lewis SC, et al. Supported Telemonitoring and Glycemic Control in People with Type 2 Diabetes: The Telescot Diabetes Pragmatic Multicenter Randomized Controlled Trial. PLoS Med 2016;13:e1002163.

69. Murad MH, Montori VM, loannidis JP, et al. How to read a systematic review and meta-analysis and apply the results to patient care: users' guides to the medical literature. JAMA 2014;312:171-9.

70. Sun X, loannidis JP, Agoritsas T, et al. How to use a subgroup analysis: users' guide to the medical literature. JAMA 2014:311:405-11.

71. Dias S, Sutton AJ, Welton NJ, et al. Heterogeneity: subgroups, metaregression, Bias and bias-adjustment. NICE Decision Support Unit Technical Support Document [Internet] 2012.

72. Health and Information and Quality Authority. Health technology assessment of chronic disease self- management support interventions, 2015.

73. Ismail K, Winkley K, Rabe-Hesketh S. Systematic review and meta-analysis of randomised controlled trials of psychological interventions to improve glycaemic control in patients with type 2 diabetes. Lancet 2004;363:1589-97.

74. Pal K, Eastwood SV, Michie S, et al. Computer-based interventions to improve self-management in adults with type 2 diabetes: a systematic review and meta-analysis. Diabetes Care 2014;37:1759-66.

75. Chrvala CA, Sherr D, Lipman RD. Diabetes self-management education for adults with type 2 diabetes mellitus: A systematic review of the effect on glycemic control. Patient Educ Couns 2016;99:926-43.

76. Norris SL, Nichols PJ, Caspersen CJ, et al. The effectiveness of disease and case management for people with diabetes. A systematic review. Am J Prev Med 2002;22(4 Suppl):15-38.
77. Glazier RH, Bajcar J, Kennie NR, et al. A systematic review of interventions to improve diabetes care in socially disadvantaged populations. Diabetes Care 2006;29:1675-88.

78. Saxena S, Misra T, Car J, et al. Systematic review of primary healthcare interventions to improve diabetes outcomes in minority ethnic groups. J Ambul Care Manage 2007;30:218-30.

79. Wang Y, Yeo QQ, Ko Y. Economic evaluations of pharmacistmanaged services in people with diabetes mellitus: a systematic review. Diabet Med 2016;33:421-7.

80. Santschi V, Chiolero A, Paradis G, et al. Pharmacist interventions to improve cardiovascular disease risk factors in diabetes: a systematic review and meta-analysis of randomized controlled trials. Diabetes Care 2012:35:2706-17.

81. Krass I, Schieback P, Dhippayom T. Adherence to diabetes medication: a systematic review. Diabet Med 2015;32:725-37.

82. Cramer JA. A systematic review of adherence with medications for diabetes. Diabetes Care 2004;27:1218-24.

83. Vermeire E, Wens J, Van Royen P, et al. Interventions for improving adherence to treatment recommendations in people with type 2 diabetes mellitus. Cochrane Database Syst Rev 2005;2:Cd003638.

84. Farmer AJ, McSharry J, Rowbotham S, et al. Effects of interventions promoting monitoring of medication use and brief messaging on medication adherence for people with Type 2 diabetes: a systematic review of randomized trials. Diabet Med 2016;33:565-79.

85. Cleveringa FG, Gorter KJ, van den Donk M, et al. Computerized decision support systems in primary care for type 2 diabetes patients only improve patients' outcomes when combined with feedback on performance and case management: a systematic review. Diabetes Technol Ther 2013:15:180-92.

86. Jeffery R, Iserman E, Haynes RB; CDSS Systematic Review Team. Can computerized clinical decision support systems improve diabetes management? A systematic review and meta-analysis. Diabet Med 2013;30:739-45. 